\title{
Tintininos (Ciliophora, Tintinnina) de águas subtropicais na região Sueste-Sul do Brasil. II. Famílias Dictyocystidae, Rhabdonellidae, Tintinnidae e Xystonellidae ${ }^{1}$
}

\author{
Luciano Felício Fernandes ${ }^{2}$ \\ ${ }^{1}$ Contribuição número 1468 do Departamento de Zoologia, Universidade Federal do Paraná. \\ 2 Departamento de Botânica, Setor de Ciências Biológicas, Universidade Federal do Paraná. Centro Politécnico, Caixa Postal \\ 19031, 81531-980 Curitiba, Paraná, Brasil. E-mail: Iff@ufpr.br
}

\begin{abstract}
Tintinnids (Ciliophora, Tintinnina) from subtropical waters of the Southern Brazil. II. Families Dictyocystidae, Rhabdonellidae, Tintinnidae and Xystonellidae. This study follows a previous work on the taxonomic survey on tintinnids (Suborder Tintinnina) collected in subtropical waters of the Southern Brazil $\left(22^{\circ} \mathrm{S}-34^{\circ} \mathrm{S}\right)$. Samples were based in different oceanographic cruises covering shelf and oceanic waters. Here, the species belonging to Families Dictyocystidae, Rhabdonellidae, Tintinnidae and Xystonellidae were described, and a general discussion regarding the tintinnid biogeography and the vertical distribution observed in some of the oceanographic cruises were carried out. For all of species the descriptions, measurements and drawings, as well comments on the taxonomy and results on horizontal and vertical distributions on the water column were furnished. The species richness was high (87 infrageneric taxa), and some species like Climacocylis scalaria, Eutintinnus spp., Salpingacantha spp., Salpingella spp. and Steenstrupiella gracilis were not previously recorded in the region. Nineteen species are new records for the Southwestern Atlantic Ocean.

KEY WORDS. Microzooplankton, Southwestern Atlantic Ocean, taxonomy.
\end{abstract}

RESUMO. Este estudo segue um trabalho prévio sobre o levantamento taxonômico dos tintininos (Subordem Tintinnina) coletados em águas subtropicais do sul do Brasil $\left(22^{\circ} \mathrm{S}-34^{\circ} \mathrm{S}\right)$. As amostras foram baseadas em diferentes cruzeiros oceanográficos cobrindo águas de plataforma e oceano. As espécies pertencentes às Famílias Dictyocystidae, Rhabdonellidae, Tintinnidae e Xystonellidae foram descritas, e uma discussão geral referente à biogeografia de tintininos e à distribuição vertical na coluna de água observada em cruzeiros oceanográficos foi realizada. Para todas as espécies foram fornecidos descrições, medidas e desenhos, bem como comentários sobre a taxonomia e resultados sobre as distribuições horizontal e vertical na coluna de água. A riqueza em espécies foi elevada (87 táxons infragenéricos), e algumas das espécies como Climacocylis scalaria, Eutintinnus spp., Salpingacantha spp., Salpingella spp. e Steenstrupiella gracilis, não haviam sido registradas na região anteriormente. Dezenove species são novos registros para o Oceano Atlântico Sul Ocidental.

PALAVRAS CHAVE. Microzooplâncton, Oceano Atlântico Sul Ocidental, taxonomia.

O presente trabalho é a continuação do estudo taxonômico sobre os tintininos iniciado em Fernandes $(1999,2004)$, e que compreende uma série de investigações destinadas a estudar a taxonomia, biogeografia e biomassa dos tintininos no Oceano Atlântico Sul-Ocidental. As amostras foram coletadas em águas subtropicais, subantárticas e antárticas em diferentes cruzeiros oceanográficos promovidos pela Marinha do Brasil e Instituto Oceanográfico da Universidade de São Paulo.

No presente trabalho foi realizado um estudo taxonômico das espécies pertencentes às Famílias Dictyocystidae, Rhabdonellidae, Tintinnidae e Xystonellidae, encontradas em águas subtropicais da plataforma e área oceânica da região SuesteSul do Brasil, coletadas durante quatro campanhas oceanográficas promovidas por diferentes instituições de pesquisa do Brasil (v. abaixo). Para as quatro famílias consideradas, são fornecidas as descrições, medidas, desenhos e fotomicrografias das espécies, bem como comentários sobre a distribuição geográfica dos táxons determinados. Uma discussão geral abrangendo as espécies determinadas nos dois trabalhos foi realizada. As famílias restantes estão contempladas em trabalho anterior (FERNANDES 2004), onde poderão ser encontrados os detalhes sobre o material coletado e a metodologia utilizada.

\section{MATERIAL E MÉTODOS}

O material é proveniente de quatro campanhas oceanográficas: Sueste IV (1984) e Monitor IV (1989), promovidas pela Diretoria de Hidrografia e Navegação da Marinha do Brasil; PROANTAR XI (1992), na primeira fase do Programa Antártico Brasileiro em novembro; e Projeto COROAS, organizado pelo Instituto Oceanográfico da Universidade de São Paulo. As amos- 
tras foram obtidas em profundidades variáveis e épocas diferentes através de arrastos verticais de rede de plâncton com $25 \mu \mathrm{m}$ de abertura das malhas, ou por filtração reversa (DoDSON \& THOMAS 1978). As características de cada coleção, as coordenadas geográficas das estações, dados físicos e químicos da água e de clorofila-a estão detalhados em trabalho anterior (FERNANDEs 2004).

O enquadramento taxonômico até o nível de Subordem baseou-se no Sistema de Levine et al. (1980), e a separação em famílias em Kofoid \& CAMpbell (1939). As abreviações realizadas no texto referente às Medidas são: indivíduos (ind.), comprimento (comp.), diâmetro oral (D.O.) e largura (larg.). As referências citadas logo abaixo dos nomes científicos referemse primeiro àquela da descrição original e, em seguida, àquelas utilizadas para a identificação das espécies.

\section{Enquadramento taxonômico das espécies}

Filo Ciliophora Doflein, 1901

Classe Polihymenophorea Jankowski, 1967

Subclasse Spirotrichia Bütschli, 1889

Ordem Oligotrichida Bütschli, 1887

Subordem Tintinnina Kofoid \& Campbell, 1929

Dictyocystidae Kent, 1881 emend. Kofoid \& Campbell, 1929

Dictyocysta Ehrenberg, 1854 emend. Kofoid \& Campbell, 1929

Dictyocysta californiensis Kofoid \& Campbell, 1929

Dictyocysta elegans lepida (Ehrenberg, 1854) Balech, 1959

Dictyocysta elegans speciosa Jörgensen, 1924

Dictyocysta mitra Haeckel, 1873

Rhabdonellidae Kofoid \& Campbell, 1929

Rhabdonella Brandt, 1906 emend. Kofoid \& Campbell, 1929 Rhabdonella cornucopia Kofoid \& Campbell, 1929

Rhabdonella elegans Jörgensen, 1924

Rhabdonella indica Laackmann, 1910

Rhabdonellopsis Kofoid \& Campbell, 1929

Rhabdonellopsis apophysata (Cleve, 1900) Kofoid \& Campbell, 1929

Tintinnidae Kofoid \& Campbell, 1929

Albatrossiella Kofoid \& Campbell, 1929

Albatrossiella agassizi Kofoid \& Campbell, 1929

Amphorellopsis Kofoid \& Campbell, 1929

Amphorellopsis acuta Kofoid \& Campbell, 1929

Amphorides Strand, 1926

Amphorides amphora (Claparéde \& Lachmann, 1858)

Strand, 1926

Brandtiella Kofoid \& Campbell, 1929

Brandtiella palliata (Brandt, 1906) Kofoid \& Campbell, 1929

Dadayella Kofoid \& Campbell, 1929

Dadayella ganimedes (Entz Sr., 1884) Kofoid \& Campbell, 1929

Daturella Kofoid \& Campbell, 1929

Daturella stramonium Kofoid \& Campbell, 1929

Eutintinnus Kofoid \& Campbell, 1939

Eutintinnus apertus (Kofoid \& Campbell, 1929) Kofoid
\& Campbell, 1939

Eutintinnus birictus (Kofoid \& Campbell, 1929) Kofoid \& Campbell, 1939

Eutintinnus fraknoi (Daday, 1887) Kofoid \& Campbell, 1939

Eutintinnus latus (Jörgensen, 1924) Kofoid \& Campbell, 1939

Eutintinnus lususundae (Entz, Sr., 1885) Kofoid \& Campbell, 1939

Eutintinnus medius (Kofoid \& Campbell, 1929) Kofoid \& Campbell, 1939

Eutintinnus pacificus (Kofoid \& Campbell, 1929) Kofoid \& Campbell, 1939

Eutintinnus stramentus Kofoid \& Campbell, 1939

Eutintinnus tubulosus (Ostenfeld, 1899) Kofoid \& Campbell, 1939

Ormosella Kofoid \& Campbell, 1929

Ormosella breslaui Kofoid \& Campbell, 1929

Salpingacantha Kofoid \& Campbell, 1929

Salpingacantha unguiculata (Brandt, 1906) Kofoid \& Campbell, 1929

Salpingella Jörgensen, 1924 emend. Kofoid \& Campbell, 1939

Salpingella curta Kofoid \& Campbell, 1929

Salpingella decurtata Jörgensen, 1924

Salpingella glockentoegeri (Brandt, 1906) Kofoid \& Campbell, 1929

Salpingella laminata Kofoid \& Campbell, 1939

Salpingella subconica Kofoid \& Campbell, 1929

Steenstrupiella Kofoid \& Campbell, 1929

Steenstrupiella gracilis (Jörgensen, 1924) Kofoid \& Campbell, 1929

Steenstrupiella steenstrupii (Claparede \& Laachmann, 1858) Kofoid \& Campbell, 1929

Xystonellidae Kofoid \& Campbell, 1929 emend. Kofoid \& Campbell, 1939

Parundella Jörgensen, 1924 emend. Kofoid \& Campbell, 1929

Parundella caudata caudata (Ostenfeld, 1899) Jörgensen, 1924

Parundella pellucida (Jörgensen, 1924) Kofoid \& Campbell, 1929

Xystonella Brandt, 1906 emend. Jörgensen, 1924

Xystonella longicauda (Brandt, 1906) Brandt, 1906

Xystonella treforti (Daday, 1887) Laackmann, 1910

Xystonellopsis Jörgensen, 1924

Xystonellopsis cymatica (Brandt, 1906) Jörgensen, 1924

Xystonellopsis dahli (Brandt, 1906) Kofoid \& Campbell, 1929

Xystonellopsis dicymatica (Brandt, 1906) Kofoid \& Campbell, 1929

Xystonellopsis gaussi (Laackmann, 1910) Kofoid \& Campbell, 1929

Xystonellopsis paradoxa (Cleve, 1900) Jörgensen, 1924 


\section{RESULTADOS E COMENTÁRIOS}

Nas quatro famílias consideradas neste trabalho foram determinados 41 táxons infragenéricos distribuídos em 17 gêneros. O gênero melhor representado foi Eutintinnus, com 9 táxons infragenéricos determinados. A seguir estão descritas as espécies encontradas, bem como comentários sobre a sua taxonomia e ocorrência nas amostras.

\section{Dictyocystidae Kent, 1881 Dictyocysta californiensis Kofoid \& Campbell, 1929} Fig. 1

Dictyocysta californiensis Kofoid \& Campbell, 1929: 288, fig. 552.Balech, 1962: 67, pl. 6, figs 61-62.

Descrição: lórica hialina robusta composta por colar e cesto. Colar composto por 6 janelas quadrangulares de lados convexos, limitadas por colunas espessadas e que formam arcos, cada um com um espinho. Cesto com ombro pouco desenvolvido, e provido de uma fileira de fenestras subquadrangulares na porção anterior e outra na região mediana, de fenestras circulares ou elípticas. Na sua porção posterior o cesto apresenta pequenas fenestras circulares, envolvidas por estrias delicadas. Parede alveolada, alvéolos semiesféricos; diafragma presente.

Medidas (1 ind.): comp. $45 \mu \mathrm{m}$; larg. $35 \mu \mathrm{m}$; D.O. $29 \mu \mathrm{m}$; altura do colar $14 \mu \mathrm{m}$.

Comentários: espécie muito semelhante à $D$. extensa Kofoid \& Campbell, da qual difere pelas medidas, ausência de extremidade aboral acuminada e lórica menos regular. As dimensões encontradas no presente trabalho enquadram-se bem nas de Kofoid \& Campbell (1929) e Balech (1962).

Ocorrência nas amostras: Operação Sueste IV (estações 6598, 6599, 6602-6604, 6615, 6618); Projeto COROAS (estações 1, 7).

\section{Dictyocysta elegans lepida (Ehrenberg, 1854) Figs 2-9, 70-74}

Dictyocysta lepida Ehrenberg, 1854: 239.- Hada, 1938: 160, fig. 76. Dictyocysta elegans lepida; Balech, 1959: 29, pl. 7, figs 110-116.

Descrição: lórica hialina ou aglutinante, formada por duas partes: colar com menos da metade do comprimento total, e cesto. O colar apresenta 6-8 janelas grandes quadrangulares a retangulares de lados paralelos a convexos. As janelas são limitadas por colunas espessadas providas de pequenas pontuações e alguns alvéolos elipsóides. Borda oral lisa e plana, ou discretamente ondulada. Cesto globoso com "ombros" conspícuos e diafragma, alvéolos e uma fileira de fenestras circulares grandes em sua região anterior ou mediana. Outras fenestras menores podem ocorrer, distribuídas irregularmente ou em fileiras. Em alguns indivíduos, estas fenestras eram irregulares, alcançando a região aboral. Os alvéolos são poligonais, nem sempre visíveis, e espalhados pelo colo. A parede da lórica pode apresentar-se parcial ou totalmente recoberta por cocolitos. Extremidade aboral obtusa a ligeiramente cuneada.

Medidas (22 ind.): comp. 49-65 $\mu \mathrm{m}$; larg. 36-46 $\mu \mathrm{m}$; D.O. 32-41 $\mu \mathrm{m}$; altura do colar 14-24 $\mu \mathrm{m}$; 6-8 janelas.

Comentários: espécie extremamente polimórfica, como demonstrado na discussão de BALECH (1959). Este autor lista vários sinônimos, e diferencia D. elegans var. lepida de D. elegans var. speciosa pela presença nesta última de uma fileira dupla de janelas no colar. Ainda segundo Balech (1959) é difícil determinar a variedade típica e, por isso, o autor sugere a retenção das duas variedades citadas por apresentarem caracteres mais facilmente identificáveis. A Figura 26 é mais semelhante às espécies sinonimizadas $D$. reticulata Kofoid \& Campbell e $D$. templum Haeckel (ver figuras de BRANDT 1907, KofoID \& CAMPBell 1929, HADA 1938), mostrando alvéolos circulares isodiamétricos, extremidade aboral aguda e janelas retangulares de comprimento aproximadamente igual às fenestras do cesto. HADA (1938) diferencia D. elegans var. lepida de D. occidentalis Kofoid \& Campbell pela presença, na primeira, de fenestras menores na região anterior do cesto, ausentes em D. occidentalis. Entretanto, BALECH (1959) a considerou sinônimo de D. elegans, procedimento também adotado neste trabalho; a Figura 21 representa um indivíduo mais próximo à "D. occidentalis". Portanto, as principais espécies sinonimizadas são: $D$. reticulata Kofoid \& Campbell, D. templum Haeckel, D. occidentalis Kofoid \& Campbell e D. lata Kofoid \& Campbell.

Ocorrência nas amostras: Operação Sueste IV (estações $6599,6604,6605,6609-6612$, 6617); Projeto COROAS (estações 1, 3, 5, 7, 10); PROANTAR XI (estações 4-6).

\section{Dictyocysta elegans speciosa (Jörgensen, 1924) Figs 75-77}

Dictyocysta elegans var. $a, c, d$; Brandt, 1906, pl. 2, figs 2 e 4 . Dictyocysta speciosa Jörgensen, 1924: 81-83.

Dictyocysta elegans speciosa; Balech, 1959: 28, pl. 7, figs 103-109.

Descrição: lórica hialina; colar composto por duas fileiras de janelas, a anterior com 6 janelas maiores quadrangulares, e a inferior com 8-9 janelas menores e sub-circulares. Colunas e arcos convexos com um espinho cada. Cesto em forma de cunha com ápice agudo e dilatado na porção anterior. Parede alveolada, alvéolos circulares pequenos. A metade posterior do cesto é composta por duas fileiras de fenestras circulares, circundadas por alvéolos. Na extremidade aboral também ocorrem fenestras. Diafragma provido de 12 peças.

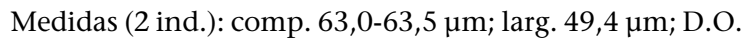
$37,0 \mu \mathrm{m}$; altura do colar $28,8 \mu \mathrm{m}$.

Comentários: indivíduos muito semelhantes às figuras de Brandt (1907, tf. 2, figs 2 e 4) como D. elegans var. mülleri e n. var. a, mais tarde consideradas por Kofoid \& CAMPBelL (1929) como D. mülleri. BALECH (1959) considera todas estas espécies como D. elegans var. speciosa. As dimensões das espécies encontradas no presente trabalho encaixam-se nas de BALECH (1959). Os exemplares de SousA \& SiLVA (1950) apresentaram as 
seguintes dimensões: comp. $50 \mu \mathrm{m}$, D.O. $38 \mu \mathrm{m}$, diâmetro maior $43,5 \mu \mathrm{m}$.

Ocorrência nas amostras: Operação Sueste IV (estações 6602, 6604, 6610, 6615, 6617) Projeto COROAS (estação 9/150-100 m).

\section{Dictyocysta mitra Haeckel, 1873 Figs 78-80}

Dictyocysta mitra Haeckel, 1873: 563, pl. 27, figs 4-5.- Balech, 1959: 27, pl. 7, figs 93-102.- Balech \& Souto, 1980: 6, fig. 5.

Descrição: lórica hialina robusta dividida em colar e cesto com diafragma, mas pouco diferenciados entre si. Colar composto por 6-7 janelas quadrangulares (maiores do que as do cesto) de vértices convexos; colunas e arcos lisos, às vezes com um espinho por arco. Cesto globoso ou em forma de projétil, totalmente perfurado por fenestras circulares a quadrangulares grandes, algumas poligonais. Extremidade aboral obtusa ou ligeiramente acuminada.

Medidas (7 ind.) comp. 59-65 $\mu \mathrm{m}$; larg. 41-42 $\mu \mathrm{m}$; D.O. 35-36 $\mu \mathrm{m}$; altura do colar 14-16 $\mu \mathrm{m}$; 6-7 janelas.

Comentários: espécie facilmente identificável devido ao cesto totalmente fenestrado e sua robustez, se comparada a outras espécies. Indivíduos como os da Fig. 78 foram bastante comuns nas amostras, diferenciando-se bastante dos encontrados na literatura. Apresentam janelas quadrangulares, cesto bem diferenciado e globoso, de parede perfurada por fenestras hexagonais bem regulares, um pouco menores na extremidade aboral, a qual é acuminada. Para lista completa de sinonímias, consulte BALECH (1959).

O exemplar das figuras 79-80 apresenta lórica bastante distinta das demais, principalmente devido a alveolação regular e composta por alvéolos de tamanho semelhante no cesto, não encontrada na literatura consultada, mas deixada aqui apenas por conveniência. As medidas são comp. 55,3-57,0 ㅆm; larg. 43-44 $\mu \mathrm{m}$; D.O. 47,5 $\mu \mathrm{m}$. As aberturas abaixo das janelas foram consideradas fenestras devido à presença de uma constrição entre as duas estruturas que deve corresponder ao limite entre o colar e o cesto. Ocorreu nas amostras do Projeto COROAS (estações 1, 7, 10).

Ocorrência nas amostras: Operação Monitor IV (estações 10/100 m, 15/100 m) Operação Sueste IV (estações 6596, 6599, 6602, 6609-661, 6606); Projeto COROAS (estações 1, 3, 5, 7, 10); PROANTAR XI (estações 5, 7, 10).

\section{Rhabdonellidae Kofoid \& Campbell, 1929 Rhabdonella cornucopia Kofoid \& Campbell, 1929} Fig. 10

Rhabdonella cornucopia Kofoid \& Campbell, 1929: 215, fig. 401.Balech, 1962: 87, pl. 10, figs 117-118.

Descrição: lórica hialina caliciforme de parede delgada, exceto na região suboral e apêndice caudal, onde é espessada. Cesto cônico, lados quase paralelos até o limite da região suboral, tornando-se então convergentes para formar um apên- dice caudal cônico de lados algo irregulares e ápice subagudo. Colar desenvolvido e liso, de lados convexos e base dilatada, circundado por um espessamento suboral triangular, onde o lado maior se estende até o limite da região suboral. Superfície da lórica provida de alvéolos e estrias curtas longitudinais, às vezes bifurcadas, difíceis de visualizar. Os alvéolos são delicados e conspícuos nas regiões oral e suboral e colar. Na região mediana os alvéolos não são visíveis (ou inexistentes?); e no apêndice caudal eles se tornam grosseiros e esparsos.

Medidas ( 2 ind.) comp. 120-135 $\mu \mathrm{m}$; larg. $50 \mu \mathrm{m}$; D.O. 43-44 $\mu \mathrm{m}$; apêndice caudal $29 \mu \mathrm{m}$.

Comentários: não foi identificado o conduto central registrado por BALECH (1962).

Ocorrência nas amostras: Operação Sueste IV (estações 6599, 6602, 6604, 6618); Projeto COROAS (estações 1, 3, $7 /$ 150-100 m, 9, 13/150-100 m, 17/130-100 m).

\section{Rhabdonella elegans Jörgensen, 1924 Figs $11-13,81$}

Rhabdonella elegans Jörgensen, 1924: 59-60.- Kofoid \& Campbell, 1929: 213, fig. 400.- Hada, 1938: 142, fig. 59.- Balech, 1962: 84, pl. 9, figs 110-114.

Descrição: lórica hialina semelhante a cálice; cesto cônico de lados nitidamente convergentes, ligeiramente convexos na metade posterior. Apêndice caudal cônico longo e afilado, de extremidade aguda. Extremidade oral provida de colar liso, cônico truncado e curto; borda suboral espessada de lados triangulares. Superfície estriada, estrias verticais ou discretamente oblíquas, algumas bifurcadas próximo à borda oral e na região mediana do cesto. As estrias percorrem inclusive o apêndice caudal, onde estão bastante comprimidas e quase unidas. Fenestras circulares pequenas e distribuídas por todo o cesto, um pouco menores na extremidade aboral. Região oral provida de alvéolos delicados subcirculares, unidos entre si.

Medidas (19 ind.) comp. 161-218 $\mu \mathrm{m}$; larg. $58 \mu \mathrm{m}$; D.O.

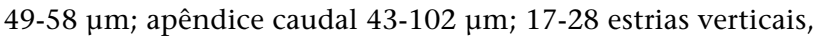
medidas a partir da região oral.

Comentários: várias espécies próximas, de difícil diferenciação e características muito tênues que separam os táxons. As descrições de Kofoid \& Campbell (1929) e Campbell (1942) estão baseadas principalmente no tamanho da borda suboral, orientação e regularidade das estrias e na morfologia do apêndice caudal, além de outros critérios pouco consistentes (aspecto geral da lórica, etc.). HADA (1938) considera várias espécies como sinônimos de $R$. elegans, tratando $R$. brandti, $R$. inflata, $R$. quantula e $R$. valdestriata como sinônimos de R. elegans. Outros autores como BALECH (1962) e Souto (1981) mantêm separadas $R$. elegans e $R$. valdestriata, mas incluem $R$. brandti em $R$. elegans. No presente trabalho, em uma amostra da Operação Monitor IV $(10 \mathrm{~m})$ foram encontrados vários indivíduos transicionais entre $R$. brandti, $R$. elegans e $R$. valdestriata. Portanto, estes táxons são aqui tratados como sinônimos de $R$. elegans Jörgensen, por ser este o nome mais antigo. 

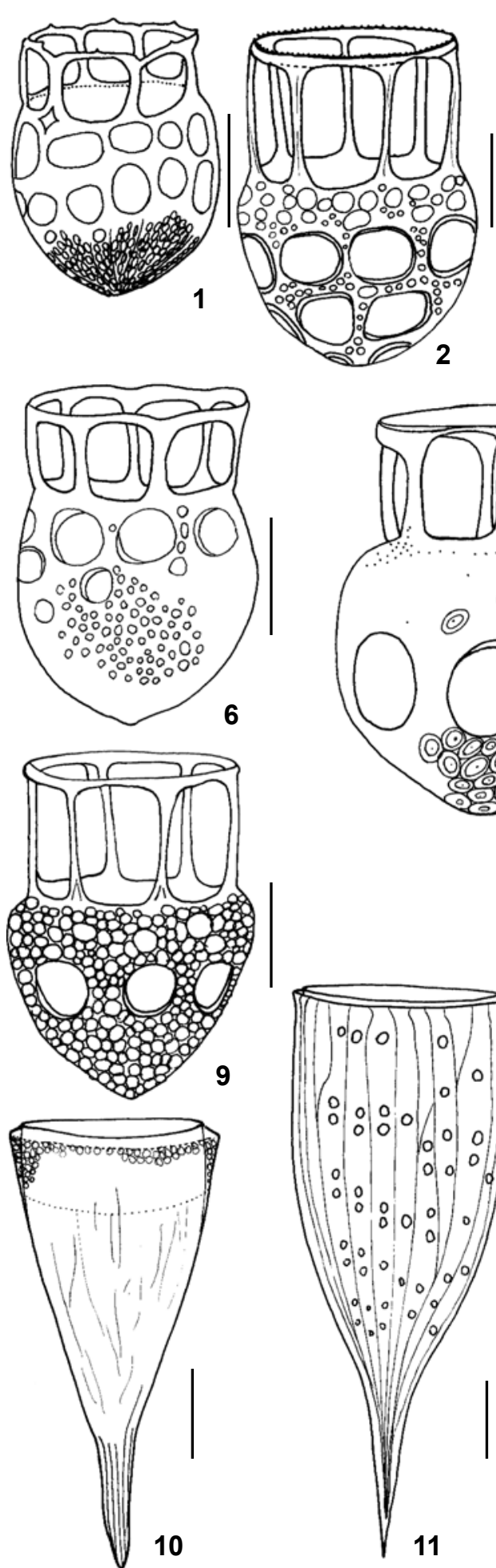

Figuras 1-15. (1) Dictyocysta californiensis; $(2,9)$ Dictyocysta elegans var. lepida; $(10)$ Rhabdonella cornucopia; $(11,13)$ Rhabdonella elegans; (14) Rhabdonella indica; (15) Rhabdonellopsis apophysata. Em 15a, detalhe da parede composta por alvéolos; em 15b, variações da base do dardo. Barras de escala $=20 \mu \mathrm{m}$.
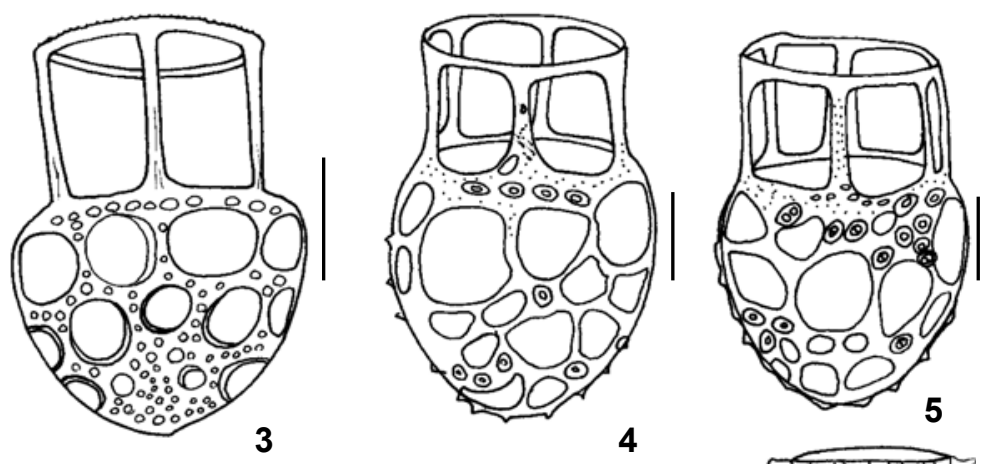

4
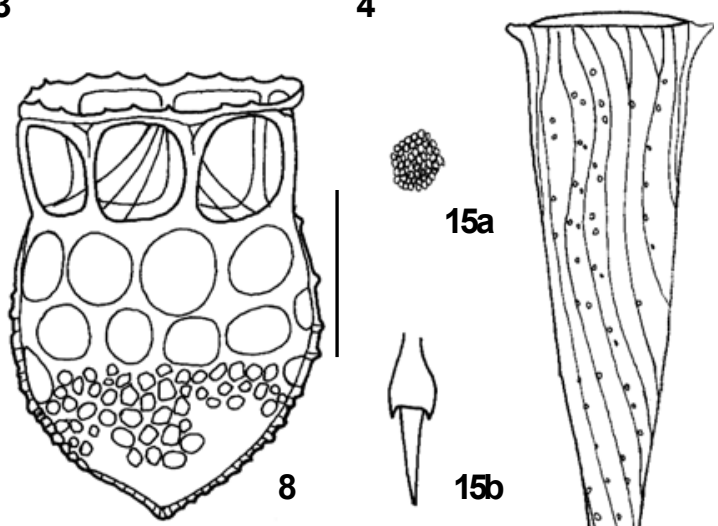

$15 a$

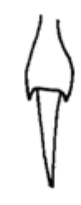

$15 b$

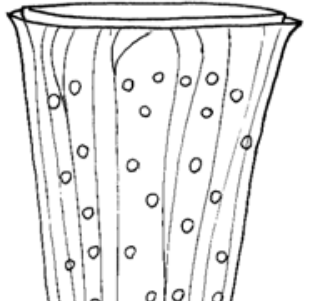

1

15

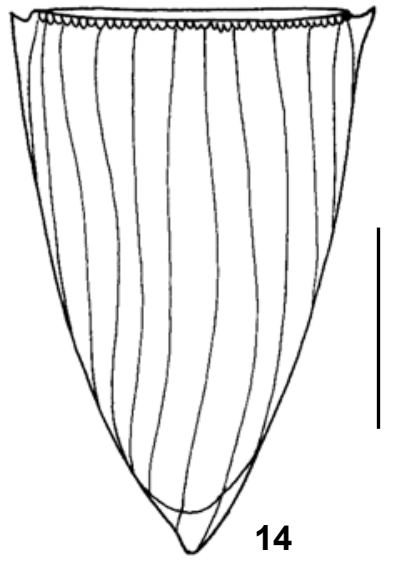

13

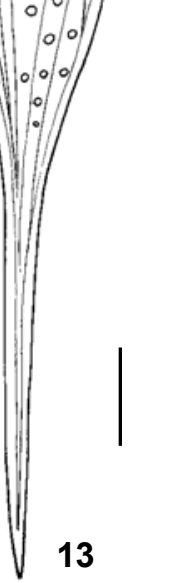

.

Revista Brasileira de Zoologia 21 (3): 605-628, setembro 2004 
Ocorrência nas amostras: Operação Monitor IV (estações 8/100 m, 10/100 m, 23/10 m); Operação Sueste IV (estações 6599, 6605, 6610-6612, 6617-6619); Projeto COROAS (estações 1, 3, 5 9, 150-100 m, 13/150-100 m, 17/130-100 m); PROANTAR XI (estações 1, 2, 5, 9).

\section{Rhabdonella indica Laackmann, 1910 Fig. 14}

Rhabdonella amor var. indica Laackmann, 1910: 461, fig. 12. Rhabdonella indica; Kofoid \& Campbell, 1929: 217, fig. 397.Balech, 1962: 82, pl. 9, figs 105-106.

Descrição: lórica hialina cônica de lados convergentes e convexos. Extremidade oral composta de anel oral curto provido de alvéolos delicados, mas conspícuos. Borda suboral espessada em forma de triângulo, onde o lado anterior (ou perpendicular) da abertura oral é côncavo, formando uma canaleta. Parede fina, exceto na região aboral, onde é bastante espessa. Superfície da lórica provida de alvéolos delicados pouco visíveis, e estrias longitudinais sinuosas, orientadas para o lado esquerdo na região oral. Extremidade aboral subaguda.

Medidas (1 indivíduo): comp. $65 \mu \mathrm{m}$; larg. $35 \mu \mathrm{m}$ (base dos espinhos); D.O. $31 \mu \mathrm{m}$; 20-26 estrias longitudinais.

Ocorrência nas amostras: Operação Sueste IV (estações 6599, 6604, 6614, 6615); Projeto COROAS (estações 1, 3).

\section{Rhabdonellopsis apophysata (Cleve, 1900) Figs 15-17, 82-83}

Cittarocylis hebe var. apophysata Cleve, 1900: 971-972, fig. 5 (à esquerda).

Rhabdonellopsis apophysata; Kofoid \& Campbell, 1929: 221/23, figs 419, 420, 424.- Balech, 1962: 88, pl. 10, figs 123-127.

Descrição: lórica hialina caliciforme de lados convergentes, formando um apêndice caudal de base alargada, do qual parte um dardo cônico curto e pontiagudo. Região oral provida de colar reduzido, o qual sobrepassa discretamente uma região suboral dilatada e convexa em seu lado externo. Esta região circunda o colar e forma um ângulo quase reto em relação à lórica. Parede bilaminar finamente alveolada; alvéolos circulares e maiores na região do cesto e menores e grosseiros no apêndice caudal. Fenestras circulares e pequenas, ocorrendo em maior número logo abaixo da região suboral até o início do apêndice caudal. A superfície apresenta estrias longitudinais sinuosas partindo da extremidade oral, tornando-se gradativamente mais próximas entre si no apêndice caudal. Na extremidade oral, muitas estrias são bifurcadas.

Medidas (52 ind.) comp. 270-320 $\mu \mathrm{m}$; larg. 62-64 $\mu \mathrm{m}$; D.O. $45-49 \mu \mathrm{m}$; comp. do espinho 25-28 $\mu \mathrm{m}$; $12-22$ estrias; $8-9$ alvéolos em $10 \mu \mathrm{m}$.

Comentários: algumas formas robustas não apresentaram dilatação suboral, ou as estrias ocorrem em menor número. A base do apêndice caudal é variável, podendo ocorrer projeções contínuas semelhantes a uma ala, ou em forma de espinho (2-4). R. intermedia Kofoid \& Campbell e R. longicaulis
Kofoid \& Campbell são muito semelhantes a $R$. apophysata, diferenciadas por Kofoid \& CAMpbell (1929) apenas pelo comprimento e número de estrias que, inclusive, se sobrepôem! Por este motivo, BALECH (1962) as considerou sinônimas de $R$. apophysata. No presente trabalho, as espécies $R$. intermedia Kofoid \& Campbell e R. triton (Zacharias) Kofoid \& Campbell, diagnosticadas por seus comprimentos, número de estrias e presença de fenestras, também foram consideradas sinônimas.

Ocorrência nas amostras: Operação Monitor IV (estações $1,2,4,8,10,10 / 10 \mathrm{~m}, 15,18,21,25 / 100 \mathrm{~m}$ ); Operação Sueste IV (estações 6598-6601, 6602-6604, 6606, 6611-6613, 66166618); Projeto COROAS (estações 1, 3, 5, 9, 10, 13/150-100 m, 17/130-100 m); PROANTAR XI (estações 1, 2, 5-15).

\section{Tintinnidae Kofoid \& Campbell, 1929 Albatrossiella agassizi Kofoid \& Campbell, 1929} Figs $18-20$

Albatrossiella agassizi Kofoid \& Campbell, 1929: 318, fig. 606.Kofoid \& Campbell, 1939: 339, pl. 29, fig. 11.

Descrição: lórica hialina; região oral (colar) em forma de cone truncado invertido; região mediana de lado paralelos, tornando-se convexos e ligeiramente divergentes, de modo a formar uma região aboral discretamente inflada. Extremidade aboral provida de um apêndice caudal que pode ser desde relativamente curto até bastante longo e afilado.

Medidas (4 ind.): comp. até o início do espinho aboral 103-107 $\mu \mathrm{m}$; larg. $13 \mu \mathrm{m}$; D.O. $22 \mu \mathrm{m}$; comp. do espinho aboral $13-64 \mu \mathrm{m}$.

Comentários: Embora KoFoId \& CAMPBell (1929) tenham encontrado apenas indivíduos de apêndice caudal longo, no presente trabalho foram identificados três indivíduos, cada um com um comprimento diferente de "espinho aboral" (13 $\mu \mathrm{m}, 28 \mu \mathrm{m}$ e $64 \mu \mathrm{m})$. A morfologia das lóricas encontradas também se assemelha à A. filigera (Laackmann), ilustrada na figura 607 de KoFoID \& CAmpbell (1929) e na figura 12 de LaAckmann (1910, Tf. 48).

Ocorrência nas amostras: Operação Monitor IV (estações 10/100 m, 14/100 m); Projeto COROAS (estações 1, 7/200-0 m).

\section{Amphorellopsis acuta (Schmidt, 1901) Figs 21,84}

Amphorella acuta Schmidt, 1901: 184-185, figs 2a-2c. Amphorellopsis acuta; Kofoid \& Campbell, 1929: 315, fig. 598.Sousa e Silva, 1954: 227, pl. 6, fig. 18.

Descrição: lórica hialina provida de uma região oral semicônica de lados retos, ou seja, a extremidade oral é mais dilatada. Cesto quase cilíndrico no terço anterior, tornando-se discretamente inflado no restante; apresenta 1-2 cristas longitudinais discretas, desde o início do cesto até atingir a extremidade aboral. Região aboral cônica de lados convexos e assimétricos. Extremidade aboral aguda. Parede delicada, lisa na região oral, formando um espessamento discreto na constrição que limita a região oral e o cesto. 


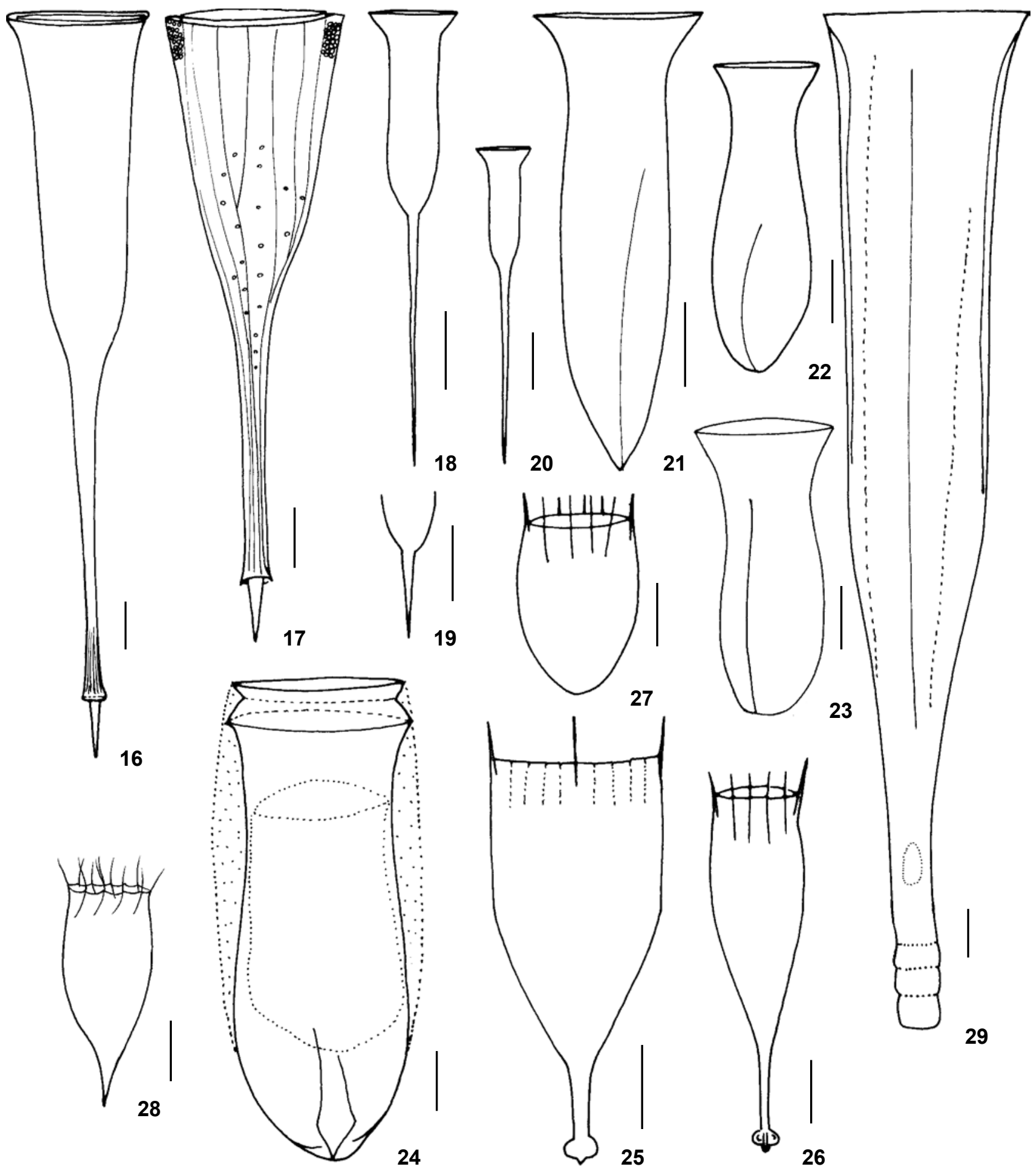

Figuras 16-29. (16, 17) Rhabdonellopsis apophysata; (18-20) Albatrossiella agassizi; (21) Amphorellopsis acuta; (22, 23) Amphorides amphora; (24) Brandtiella palliata; $(25,28)$ Dadayella ganimedes, note o polimorfismo das lóricas; $(29)$ Daturella stramonium. Barras de escala $=20 \mu \mathrm{m}$. 
Medidas (6 ind.): comp. 102-115 $\mu \mathrm{m}$; D.O. 39-42 $\mu \mathrm{m}$; larg. maior no cesto $24-27 \mu \mathrm{m}$.

Comentários: encontrada apenas por SASSI \& Melo (1989) em águas do nordeste do Brasil.

Ocorrência nas amostras: PROANTAR XI (estações 18,19).

\section{Amphorides amphora (Claparéde \& Lachmann, 1858)} Figs 22-23

Amphorella amphora Claparéde \& Lachmann, 1858: 199, pl. 8, fig. 3.- Balech, 1959: 54, pl. 20, figs 303-305.

Amphorides amphora; Strand, 1926: 30.- Souto, 1970b: 200, fig. 41.

Descrição: lórica hialina, região oral cônica truncada e invertida, provida de espessamento da parede do colar que se acentua próximo à constrição. Cesto cilíndrico de lados convexos e inflados na região aboral. Extremidade aboral obtusa, discretamente rostrada, portando crista que se estende até a região mediana ou na constrição da lórica.

Medidas (18 ind.): comp. 121-138 $\mu \mathrm{m}$; D.O. 43-44 $\mu \mathrm{m}$; larg. 45-46 $\mu \mathrm{m}$.

Ocorrência nas amostras: Operação Monitor IV (estações 4,6/10 m, 12/10 m, 23); Operação Sueste IV (estações 6618, 6610); Projeto COROAS (estações 1, 3, 5, 10); PROANTAR XI (estações 3, 4, 8-18).

\section{Brandtiella palliata (Brandt, 1906)}

Fig. 24

Tintinnus palliatus Brandt, 1906, pl. 70, fig. 1.- Brandt, 1907: 33. Brandtiella palliata; Kofoid \& Campbell, 1929: 325, fig. 623.Souto, 1981, fig. 170, n. 124.

Descrição: lórica hialina delicada, composta por colar dividido em um anel suboral e uma prega inferior, de modo que os lados aparecem projetados e triangulares. Cesto de paredes convergentes na região anterior, ligeiramente constrita, tornando-se dilatado até a região aboral, a qual apresenta-se cônica de lados convexos e extremidade obtusa a acuminada. A região aboral possui duas estrias formando uma figura losangular. A lórica é revestida por mucilagem hialina, pouco conspícua.

Medidas (3 ind.): comp. 158-160 $\mu \mathrm{m}$; D.O. $52 \mu \mathrm{m}$.

Comentários: Lórica bastante transparente, de difícil visualização ao microscópio ótico. A espécie não identificada em Thompson et al. (1999, Pl. 3, Fig. 64) é sem dúvida B. palliata.

Ocorrência nas amostras: Operação Sueste IV (estações 66026604, 6615, 6616); Projeto COROAS (estações 7, 9/150-100 m).

\section{Dadayella ganimedes (Entz, Sr., 1884) Figs 25-28}

Tintinnus ganimedes Entz, Sr., 1884: 409, pl. 24, figs 17-18. Dadayella ganimedes; Hada, 1938: 171, fig. 86.- Sousa \& Silva, 1954: 228, pl. 7, figs 1-2; pl. 7, figs 3-9.- Sassi \& Melo, 1986: 5, figs 35-39.

Descrição: lórica hialina em forma de cálice alongado, de lados convexos até metade da lórica, tornando-se convergentes para formar um apêndice caudal cônico afilado, provido de expansão subterminal semelhante a duas alas. Próximo à região oral os lados são ligeiramente divergentes, constituindo uma constrição, a qual limita uma borda oral freqüentemente indistinta. Uma série de 4-10 linhas longitudinais e paralelas entre si, partindo da região suboral, atravessa a região oral e sobrepassa a borda oral em quase a metade do seu comprimento.

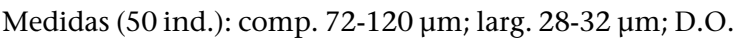
26-32 $\mu \mathrm{m}$

Comentários: espécie bastante polimórfica. Algumas formas não apresentam apêndice caudal ou a dilatação no mesmo (Figs 53-54), os lados da lórica podem ser retos ou convexos, e o número e tamanho das linhas longitudinais são variáveis. Em águas oceânicas de maior influência da Corrente do Brasil, as lóricas eram semelhantes à descrição acima. O D.O. apresenta faixa de variação relativamente ampla; alguns indivíduos aberrantes com D.O. de 39-41 $\mu$ m (Fig. 25). Devido à sua morfologia variável, várias espécies foram descritas, como $D$. acutiformis (Jörgensen), D. bulbosa (Brandt) Kofoid \& Campbell e D. curta, todas consideradas sinônimas de D. ganimedes no presente trabalho. Em Sousa \& SiLva (1954) estão ilustrados vários morfótipos da espécie, sob os nomes $D$. ganimedes e "D. bulbosa". Para lista completa de sinonímias, consulte SASsI \& Melo (1986).

Ocorrência nas amostras: Operação Monitor IV (estações 1, 2, 16, 26/10 m, 8/100 m, 10/100 m); Operação Sueste IV (estações 6597-6601, 6604-6607, 6608, 6610-6612, 6619-6620); Projeto COROAS (estações 1, 3, 5, 7, 9); PROANTAR XI (estações 1-5, 7-13, 16).

\section{Daturella stramonium Kofoid \& Campbell, 1929} Fig. 29

Daturella stramonium Kofoid \& Campbel, 1929: 346, fig. 66.-

Balech, 1962: 118, pl. 14, fig. 173.

Descrição: lórica hialina grande e alongada, semelhante à corneta. Lados quadrangulares convergentes e retos, ligeiramente convexos na região mediana, formando uma dilatação. Margem oral com uma dobra da parede formando prega, seguida por colar cônico-truncado de lados convexos. Parede provida de cristas longitudinais evidentes e torcidas, a partir da região suboral estendendo-se à região aboral. Região aboral provida de um a três anéis posteriores de lados convexos e uma grande fenestra elíptica pouco acima dos anéis; extremidade aboral aberta.

Medidas ( 2 ind.): comp. 158-162 $\mu \mathrm{m}$, D.O. $84 \mu \mathrm{m}$, diâmetro aboral $20 \mu \mathrm{m}$, altura do colo $20 \mu \mathrm{m}$.

Ocorrência nas amostras: Operação Sueste IV (estações 6602, 6604, 6615, 6616); Projeto COROAS (estação 1/200-0 m).

\section{Eutintinnus apertus (Kofoid \& Campbell, 1929)}

\section{Fig. 30}

Tintinnus apertus Kofoid \& Campbell, 1929: 331, fig. 647.

Eutintinnus apertus; Kofoid \& Campbell, 1939: 363, fig. 4.-

Balech, 1959: 58, pl. 21, fig. 321, pl. 22, fig. 322.

Descrição: lórica hialina pequena, de lados discretamen- 
te convergentes no quarto anterior (região oral) e paralelos até a região aboral, quando se tornam convexos e convergentes e, na borda aboral retomam a orientação paralela. Também é comum a lórica de lados paralelos desde a borda oral até metade ou dois terços de sua extensão, quando se tornam convergentes e retos. Bordas oral e aboral lisas, sem ornamentações.

Medidas (13 ind.) comp. 72-106 $\mu \mathrm{m}$; larg. $37 \mu \mathrm{m}$; D.O. $36 \mu \mathrm{m}$; diâmetro aboral 12-21 $\mu \mathrm{m}$.

Comentários: lórica polimórfica, protozoário geralmente ocupando 50-60\% do volume interno da lórica. Presença comum de 2-4 células de Chaetoceros dadayi fixos à parede anterior, e próximo à borda oral. As ilustrações e dimensões de Balech (1959) e Kofoid \& Campbell (1939) correspondem melhor ao nosso material.

Ocorrência nas amostras: Operação Monitor IV (estações 21/100 m, 5/100 m); Operação Sueste IV (estações 6596, 6597, 6602, 6604-6606, 6608-66116618, 6621); Projeto COROAS (estações 1, 3); PROANTAR XI (estações 1-7, 9-12).

\section{Eutintinnus birictus (Kofoid \& Campbell, 1929)}

\section{Figs $31-32$}

Tintinnus birictus Kofoid \& Campbell, 1929: 332, fig. 634. Eutintinnus birictus; Kofoid \& Campbell, 1939: 364, pl. 31, fig.

9.- Hada, 1938: 178, fig. 94.- Souto 1970b: 203, fig. 48 .

Descrição: lórica hialina grande; região oral semelhante a cone truncado invertido de lados côncavos, em seguida tornando-se discretamente convergentes até a região aboral. Esta é um cone truncado de lados ligeiramente côncavos ou retos, menores do que na região oral.

Medidas (4 ind.) comp. 180-190 $\mu \mathrm{m}$; larg. na região mediana 23-25 $\mu \mathrm{m}$; D.O. $41 \mu \mathrm{m}$; diâmetro aboral $32 \mu \mathrm{m}$.

Comentários: lóricas semelhantes às de $E$. fraknoi. Os critérios para separação das duas espécies (exclusivamente para nossos exemplares) foram: E. birictus apresenta lórica mais curta e larga, com a região aboral mais desenvolvida do que em $E$. fraknoi. Esta espécie é mais delgada em relação ao comprimento, e a região aboral é um cone de pequena altura (ou seja, lados pouco desenvolvidos). E. fraknoi apresenta dilatação da borda oral, inexistente em E. birictus. Nas amostras analisadas, as duas espécies (E. birictus e $E$. fraknoi) foram encontradas juntas. Entretanto, puderam ser diferenciadas pela variação de tamanho bastante delimitada, com E. birictus (<190 $\mu \mathrm{m})$ sempre menor do que $E$. fraknoi $(>270 \mu \mathrm{m})$. Os exemplares de BALECH (1962) são bem maiores (E. birictus: comp. 384-527 $\mu \mathrm{m}$, D.O. 66-70 $\mu \mathrm{m}$; E. fraknoi: comp. 263-495 $\mu \mathrm{m}$, D.O. 54-62 $\mu \mathrm{m})$. BALECH (1959) registra indivíduos da espécie E. fraknoi com D.O. 54-63 $\mu \mathrm{m}$, mas geralmente entre 58 e $60 \mu \mathrm{m}$, medidas bem superiores a encontrada no presente trabalho $(41 \mu \mathrm{m})$. A confusão sugere seguir o procedimento de HADA (1938), unindo as duas espécies e mantendo $E$. fraknoi por ser o nome mais antigo.

Ocorrência nas amostras: Operação Monitor IV (estação 10/100 m); Operação Sueste IV (estações 6618, 6619); Projeto COROAS (estações 1, 7, 10); PROANTAR XI (estações 4-7, 9).

\section{Eutintinnus fraknoii (Daday, 1887) Figs 33-36}

Tintinnus fraknóii [sic] Daday, 1887: 526, 528, 530, pl. 18, fig. 1.- Hada, 1938: 177, fig. 98 (discussão detalhada de espécies próximas).

Eutintinnus fraknoii; Kofoid \& Campbell, 1939: 367, pl. 31, fig. 10.- Souto, 1970a: 223, fig. 10.

Descrição: lórica hialina grande, região oral semelhante a um cone truncado invertido de lados convergentes e discretamente côncavos, tornando-se quase paralelos até a região aboral. Em algumas lóricas os lados tornam-se paralelos um pouco antes da região aboral. Esta é um cone truncado de lados retos, e bem menor do que a região oral. Borda oral dilatada, projetada para fora em ângulo reto em relação ao comprimento da lórica.

Medidas (9 ind.) comp. 328-410 $\mu \mathrm{m}$; larg. média $24 \mu \mathrm{m}$;

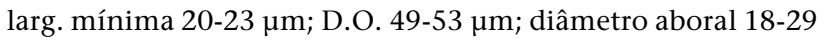
$\mu \mathrm{m}$. Uma lórica apresentou as medidas: comp. $270 \mu \mathrm{m}$; D.O. 49 m; diâmetro aboral $22 \mu \mathrm{m}$.

Comentários: veja em $E$. birictus.

Ocorrência nas amostras: Operação Monitor IV (estações 10/10 m, 22/100 m); Operação Sueste IV (estações 6606-6609, 661, 6619, 6621); Projeto COROAS (estações 1, 10, 19/130-70 $\mathrm{m})$; PROANTAR XI (estações 4-6, 10, 11).

\section{Eutintinnus latus (Jörgensen, 1924) Fig. 37}

Tintinnus fraknóii [sic] var. latus Jörgensen, 1924: 11-12, fig. 6. Eutintinnus latus; Kofoid \& Campbell, 1939: 362-364.- Balech, 1962: 115, pl. 15, fig. 200.

Descrição: lórica hialina grande pardo-amarelada; parede dupla conspícua, mais robusta do que em outras espécies do gênero. Bordas oral e aboral dilatadas transversalmente, principalmente esta última. Região oral expandida como um cone truncado, lados côncavos e convergentes até a porção posterior, quando se dilatam novamente para formar a região aboral. Esta apresenta diâmetro aboral bastante próximo ao da extremidade oral. Na superfície da lórica ocorrem pontuações muito pequenas, nem sempre visíveis, e pequenas estrias próximas à região oral.

Medidas (4 ind.) comp. 342-370 $\mu \mathrm{m}$; D.O. $67 \mu \mathrm{m}$; diâmetro aboral 54-56 $\mu \mathrm{m}$.

Comentários: medidas semelhantes às de BALECH (1962), para o Oceano Pacífico. Protozoário ocupando 33-40\% do volume da lórica, e localizado em sua porção mediana. A lórica é bastante espessa, contrastando com as outras espécies encontradas neste trabalho. HADA (1938) considerou E. latus como sinônimo de $E$. fraknoi, mas BALECH (1962) não concordou e manteve a espécie independente. Em nosso material E. latus apresentou características distintas das espécies "próximas" de Hada.

Ocorrência nas amostras: Operação Monitor IV (estação 10/100 m) Operação Sueste IV (estações 6602-6604).

Revista Brasileira de Zoologia 21 (3): 605-628, setembro 2004 


\section{Eutintinnus Iususundae (Entz, Sr., 1885) Figs 38-39}

Tintinnus lusus-undae [sic] Entz, Sr., 1885: 202, pl. 14, fig. 12.Hada, 1938: 173, fig. 88 (v. os comentários para outras espécies).

Eutintinnus lususundae; Kofoid \& Campbell, 1939: 368, pl. 32, fig. 3.- Balech, 1959: 59, pl. 22, fig. 323.- Balech, 1962: 116 (para discussão em E. medius).

Descrição: lórica hialina cônica truncada e alargada, transparente ou pardo-amarelada. Região oral expandida, de lados côncavos, borda oral ligeiramente espessada. Lados convergentes, quase paralelos entre si; extremidade aboral discretamente inflexionada ou reta, ou seja, dilatação aboral inexistente. Superfície externa da lórica provida de pequeníssimas granulações próximas umas ás outras.

Medidas (38 ind.) comp. 203-380 $\mu \mathrm{m}$; D.O. 48-51 $\mu \mathrm{m}$; larg. região mediana 23-38 $\mu \mathrm{m}$; diâmetro aboral 25-30 $\mu \mathrm{m}$.

Comentários: espécie semelhante à E. tenuis e E. fraknoi, das quais difere por apresentar uma pequena inflexão na extremidade aboral; nas outras duas há uma pequena expansão (Balech 1962). Hada (1938) considerou E. tenuis sinônima de E. lususundae var. tenuis Kofoid \& Campbell. Outras espécies próximas são E. medius Kofoid \& Campbell e E. elegans (Jörgensen), ambas diferindo de E. lususundae por apresentarem região aboral expandida, ainda que discretamente. Entretanto, BALECH (1962) fornece duas ilustrações (figs 191, 192) onde não se observa expansão aboral. Todas estas espécies apresentam D.O. semelhante, embora seus comprimentos possam variar bastante, particularmente em relação à $E$. fraknoi (310-420 $\mu \mathrm{m})$. Portanto, a identificação deste grupo é bastante complicada e, no presente trabalho, consideramos E. fraknoi, E. lususundae e E. medius independentes, baseando-se na região aboral e no comprimento. Convém lembrar que Souto (1970b) e AlDER (1999) encontraram uma espécie determinada como E. medius, e a registraram como a espécie dominante dentro do gênero Eutintinnus na Água Subtropical do Brasil. Entretanto, as ilustrações de Souto $(1970 b)$ diferem muito das nossas e de Kofoid \& CAmpbell $(1929,1939)$.

A tendência a uma pequena convergência dos lados da lórica na extremidade aboral é denominada "inflexão" por Balech \& Souto (1980), como ocorre em E. lususundae.

Ocorrência nas amostras: espécie do Gênero Eutintinnus mais abundante na região; PROANTAR XI (estações 2, 4-18); Projeto COROAS (estações 1, 10).

\section{Eutintinnus medius (Kofoid \& Campbell, 1929) Figs 40,85}

Tintinnus medius Kofoid \& Campbell, 1929: 336, fig. 629. Eutintinnus medius; Kofoid \& Campbell, 1939: 369; pl. 31, fig. 8.- Balech, 1962: 116; pl. 15, figs 190-192.

Descrição: lórica hialina cônica truncada e alargada, transparente. Região oral expandida, de lados côncavos, borda oral ligeiramente espessada. Lados convergentes, quase paralelos entre si; extremidade aboral discretamente expandida para fora, ou seja, com dilatação aboral. Lados discretamente inflados no terço posterior da lórica.

Medidas (26 ind.) comp. 138-167 $\mu \mathrm{m}$; D.O. $42-48 \mu \mathrm{m}$; larg. região mediana 29-35 $\mu \mathrm{m}$; diâmetro aboral 23-32 $\mu \mathrm{m}$.

Comentários: na Fig. 85 está mostrada uma lórica com dilatação aboral muito discreta, sugerindo a sobreposição de características diagnósticas entre E. medius e E. lususundae. Para detalhes, veja os comentários em Eutintinnus lususundae.

Ocorrência nas amostras: espécie abundante dentro do Gênero Eutintinnus. Operação Monitor IV (estações 1, 2, 18); PROANTAR XI (estações 1-7, 10-16, 23-25).

\section{Eutintinnus pacificus (Kofoid \& Campbell, 1929)}

\section{Fig. 41}

Tintinnus pacificus Kofoid \& Campbell, 1929: 337, fig. 632.

Eutintinnus pacificus; Kofoid \& Campbell, 1939: 337, figs 2, 3.

Descrição: lórica hialina pequena, lados paralelos desde a borda oral até metade ou dois terços de sua extensão, quando se tornam convergentes e retos. Bordas oral e aboral lisas, sem ornamentações.

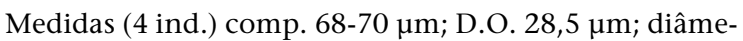
tro aboral $19 \mu \mathrm{m}$.

Comentários: espécie muito semelhante na forma geral à E. apertus (Kofoid \& Campbell) Kofoid \& Campbell e E. pinguis (Kofoid \& Campbell) Kofoid \& Campbell. Ela difere da primeira apenas pela terminação da região aboral e dimensões um pouco menores, gerando dúvidas quanto à determinação. Em E. pinguis as dimensões são maiores (comp. 114-161 $\mu \mathrm{m}$, D.O. 41-47 $\mu \mathrm{m}$ ) (Kofoid \& CAMPBell 1939).

Ocorrência nas amostras: PROANTAR XI (estações 10, 11)

\section{Eutintinnus stramentus (Kofoid \& Campbell, 1929)}

Fig. 42

Tintinnus stramentus Kofoid \& Campbell, 1929: 339, fig. 635.Hada, 1938: 176, fig. 92.

Eutintinnus stramentus; Kofoid \& Campbell, 1939: 373, pl. 32, fig. 6.- Souto, 1970a: 223, fig. 9.

Descrição: lórica hialina cônica truncada e alongada, parede delicada de lados convergentes desde a região oral, tornando-se quase paralelos a partir da porção mediana. Borda oral discretamente espessada para o exterior, ou seja, em direção transversal ao comprimento da lórica. Região aboral não alargada, sem inflexão.

Medidas ( 2 ind.) comp. 208-210 $\mu \mathrm{m}$; larg. média $20 \mu \mathrm{m}$; D.O. $29 \mu \mathrm{m}$; diâmetro aboral $10 \mu \mathrm{m}$.

Ocorrência nas amostras: Operação Monitor IV (estações 8/10 m, 12/100 m); Projeto COROAS (estações 1, 3, 13/150100 m); PROANTAR XI (estação 3). 


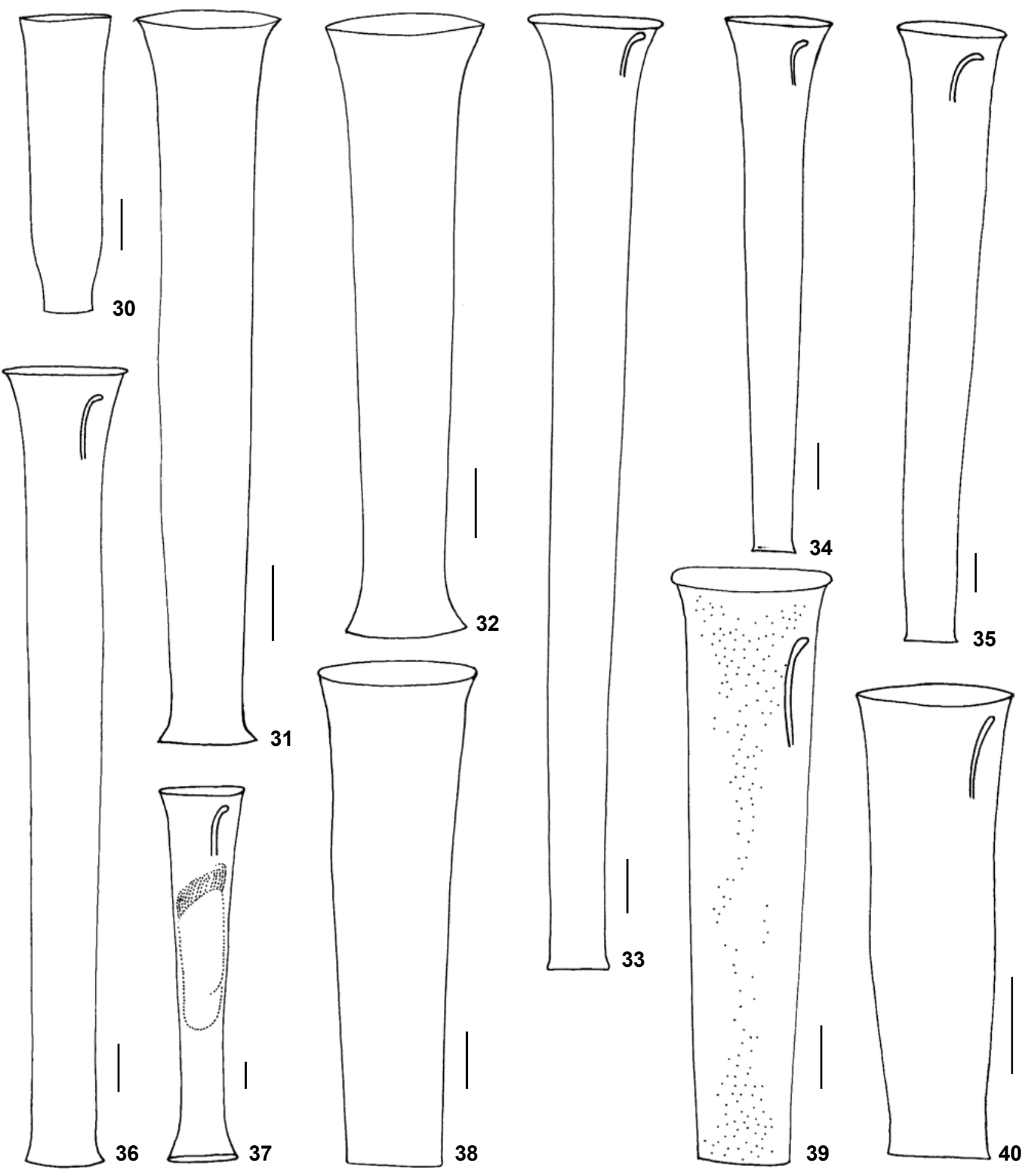

Figuras 30-40. (30) Eutintinnus apertus; $(31,32)$ Eutintinnus birictus; $(33,36)$ Euntintinnus fraknoi; $(37)$ Eutintinnus latus; $(38,39)$ Eutintinnus lususundae; (40) Eutintinnus medius. No interior de algumas lóricas estão mostrados desenhos "em corte" das margens orais, para observação das expansões orais. Barras de escala $=20 \mu \mathrm{m}$. 


\section{Eutintinnus tubulosus (Ostenfeld, 1899) Figs 43-44}

Tintinnus tubulosus Ostenfeld, 1899: 439, fig. 2f.

Eutintinnus tubulosus; Kofoid \& Campbell, 1939: 374, pl. 32,

fig. 8.- Souto, 1970b: 207, fig. 58.

Descrição: lórica hialina em forma de cone truncado e invertido, aproximadamente cilíndrica, ou seja, lados quase paralelos entre si. A margem oral apresenta um discreto rebordo oral para cima. Não há expansões oral e aboral, bordas espessadas ou inflexão aboral.

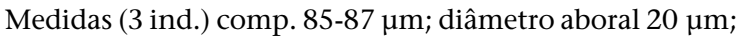
D.O. $29 \mu \mathrm{m}$.

Ocorrência nas amostras: PROANTAR XI (estações 4, 11, 12)

\section{Ormosella breslaui Kofoid \& Campbell, 1929 Figs 45, 86}

Ormosella breslaui Kofoid \& Campbell, 1929: 323, fig. 616.Balech, 1968: 189, pl. 4, figs 39-40.

Descrição: lórica hialina provida de colar desenvolvido e cônico truncado de lados retos e espessados até a base. Base reta, formando uma plataforma, seguida por uma constrição. Corpo de parede espessa, lados côncavos ou retos no terço anterior tornando-se convexos, convergindo a partir da região mediana da lórica para formar a região aboral ogival. Extremidade oral composta por apêndice caudal cônico e pontiagudo. A parede da lórica apresenta estrias longitudinais tênues, desde a extremidade aboral até a base da plataforma, não atingindo o colar.

Medidas (5 ind.) comp. $58 \mu \mathrm{m}$, D.O. 22-35 $\mu \mathrm{m}$ (a maioria maior do que $34 \mu \mathrm{m}$ ), larg. $17 \mu \mathrm{m}$, apêndice caudal 8-12 $\mu \mathrm{m}$ (alguns com $5 \mu \mathrm{m}$ ).

Comentários: algumas lóricas apresentaram morfologia semelhante à O. apsteinii Kofoid \& Campbell (1929: 323, fig.620), mas suas medidas foram menores e dentro das atribuídas à O. breslaui. Em O. haeckeli Kofoid \& Campbell as medidas são maiores e o apêndice caudal é curto e menos pontiagudo (ver Balech 1971, Kofoid \& Campbell 1929).

Ocorrência nas amostras: Operação Monitor IV (estação 10/100 m); Operação Sueste IV (estações 6599, 6601, 6616, 6617); Projeto COROAS (estação 9/150-100 m).

\section{Salpingacantha unguiculata (Brandt, 1906) Figs 46-50, 87}

Tintinnus undatus var. unguiculatus Brandt, 1906: 32, pl. 67, figs 2, 5, 6, 6a.- Brandt, 1907: 392.

Salpingacantha unguiculata; Kofoid \& Campbell, 1929: 358, fig. 692.- Kofoid \& Campbell, 1939, pl. 34, fig. 6.

Descrição: lórica hialina cilíndrica alongada de lados paralelos em sua maior extensão e parede muito delgada. Extremidade oral provida de 4-5 dentes triangulares curtos e paralelos à parede da lórica. Na região suboral, logo abaixo da extremidade oral, pode ocorrer uma constrição discreta ou pe- quena dilatação (Fig. 46) que se continua até a borda oral, constituindo o colar. Região aboral de lados convergentes convexos a retos, formando um cone truncado de ápice reto. As cristas aborais são oblíquas e se apresentam como alas projetadas da parede da lórica, cruzando-se em sua parte mediana e alcançando os lados na porção logo acima da região aboral do cesto. Algumas cristas podem ser simples, isto é, visualizadas como estrias oblíquas a retas não se cortando entre si.

Medidas (4 ind.) comp. 210-258 $\mu \mathrm{m}$; D.O. 11-14 $\mu \mathrm{m}$; larg. 12-14 $\mu \mathrm{m}$.

Comentários: medidas semelhantes às de KofoId \& Campbell $(1929,1939)$.

Os exemplares das Figs 50 e 87 apresentaram lóricas bastante distintas devido às dimensões, com comp. 79-82 $\mu \mathrm{m}$; D.O. 9-10 $\mu \mathrm{m}$; larg. 13-14 $\mu \mathrm{m}$, extremidade oral denteada, 4-5 dentes de lados retos a ligeiramente convergentes e região aboral provida de 5-6 cristas aborais longitudinais oblíquas convexas que se estendem por toda a sua área. Estas são bem menores do que as demais do gênero Salpingacantha, no qual o comprimento é superior a $147 \mu \mathrm{m}$, freqüentemente acima de $250 \mu \mathrm{m}$. A ocorrência nas amostras destes exemplares anômalos foi: Operação Monitor IV (estações 10/10 m, 10/100 m); Operação Sueste IV (estações 6604, 6615).

Ocorrência nas amostras: Operação Monitor IV (estação 10/100 m); Operação Sueste IV (estações 6604, 6612, 6613); PROANTAR XI (estações 8, 10, 12).

\section{Salpingella curta Kofoid \& Campbell, 1929} Fig. 88

Salpingella curta Kofoid \& Campbell, 1929: 352, fig. 675.- Kofoid \& Campbell, 1939: 383, pl. 34, figs 7, 9.

Descrição: lórica hialina pequena em forma de projétil. Colar discreto, em forma de cone truncado e invertido. Corpo cilíndrico de lados paralelos a ligeiramente divergentes no terço posterior, onde se tornam convergentes até a extremidade aboral aguda. Esta parte cônica da lórica apresenta 4-6 cristas pronunciadas. Na região suboral ocorre um diafragma composto por duas peças oblíquas. Protozoário ocupando 70-90\% da lórica.

Medidas (6 ind.) comp. 72-79 $\mu \mathrm{m}$, D.O. 17-19 $\mu \mathrm{m}$.

Comentários: dimensões menores do que em Koford \& Campbell $(1929,1939)$ e Balech (1959).

Ocorrência nas amostras: Operação Sueste IV (estações 6599, 6602-6604); Projeto COROAS (estações 1, 17/130-100 m).

\section{Salpingella decurtata Jörgensen, 1924 Figs $51-52$}

Salpingella decurtata Jörgensen, 1924: 15, fig. 10.- Kofoid \& Campbell, 1929: 352, fig. 685.- Sousa \& Silva, 1950: 22, pl. 4, fig. 14.-Balech, 1959: 53, pl. 20, figs 291-292.

Descrição: lórica hialina alongada de paredes muito finas. Colar cônico truncado invertido de lados convexos projetados para o exterior, formando uma dilatação discreta. 


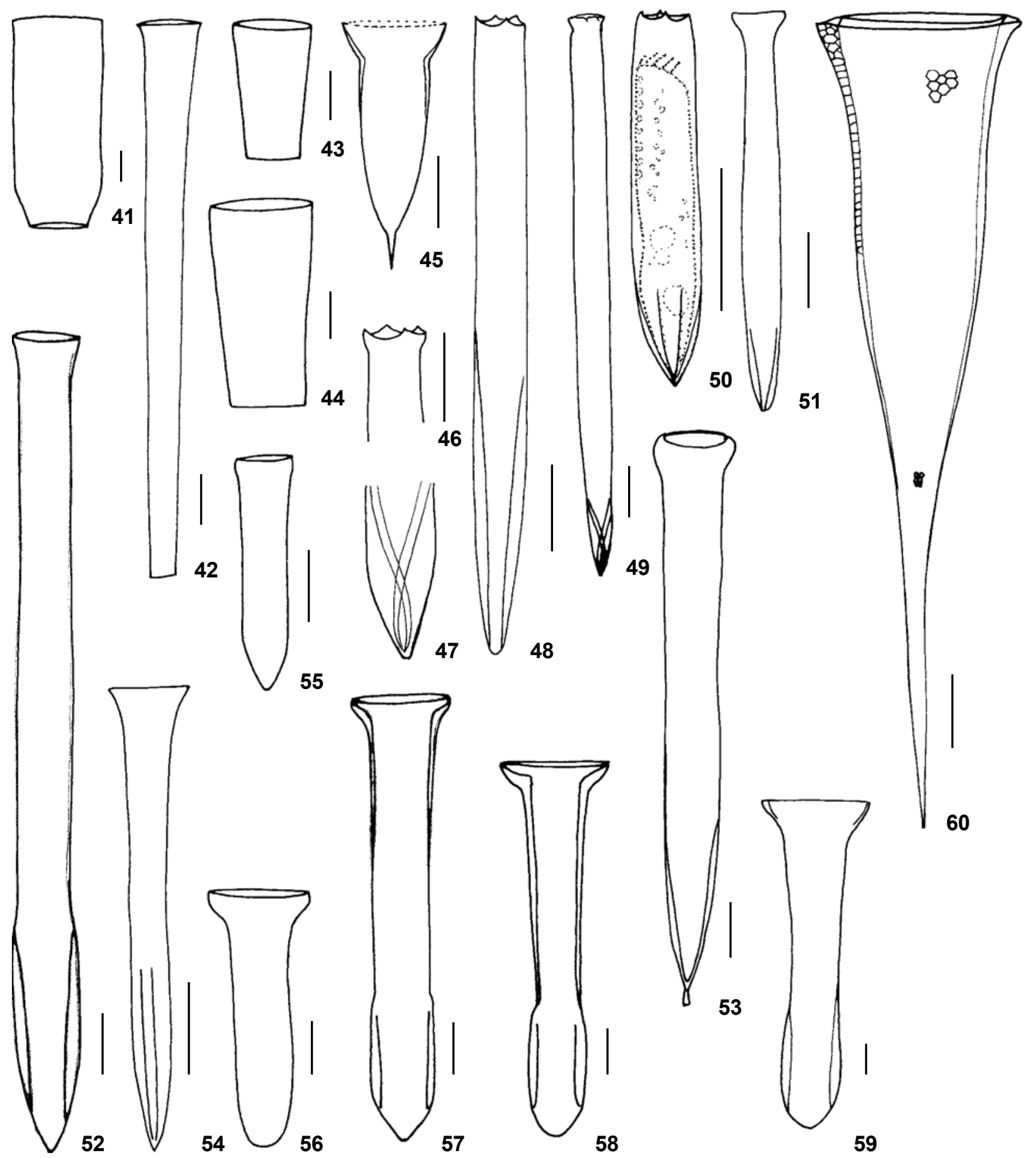

Figuras 41-60. (41) Eutintinnus pacificus; (42) Eutintinnus stramentus; $(43,44)$ Eutintinnus tubulosus; $(45)$ Ormosella bres/auii; $(46,49)$ Salpingacantha unguiculata; (50) Salpingacantha unguiculata (?); (51, 52) Salpingella decurtata; (53) Salpingella glockentoegeri; (54) Salpingella laminata; (55) Salpingella subconica; $(56)$ Steenstrupiella gracilis; $(57,59)$ Steenstrupiella steenstrupii; $(60)$ Xystonella longicauda. Barras de escala $=20 \mu \mathrm{m}$. 
Borda oral lisa, incluída na dilatação dos lados do colar. Abaixo deste, os lados são aproximadamente paralelos, tornandose convergentes na região aboral, de aspecto cônico e contendo as cristas aborais, em número de 4-5. As cristas são quase retas e partem de uma extremidade quadrangular pequena (às vezes inexistente).

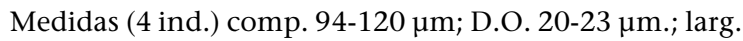
13-29 $\mu \mathrm{m}$.

Comentários: medidas de D.O. muito amplas, causando dúvidas quanto à identificação. Os exemplares de Sousa \& SiLva (1950) são um pouco maiores do que o presente material. Na ilustração de BALECH (1962) nota-se um diafragma, inexistente em nossos exemplares. Esta espécie é semelhante a $S$. subconica, diferenciadas pelas dimensões da lórica que, em nosso material, apresentaram-se bem distintas.

Ocorrência nas amostras: Operação Monitor IV (estação 9/100 m); Operação Sueste IV (estações 6602-6604).

\section{Salpingella glockentoegeri (Brandt, 1906) Figs 53,89}

Tintinnus acuminatus var. e glockentögeri Brandt, 1906: 32, pl. 67, figs 7-8, pl. 68, figs $1,5,5$ a.

Salpingella glockentoegeri; Kofoid \& Campbell, 1929: 353, fig. 688.- Balech, 1959: 52, pl. 19, figs 284-290.- Balech \& Souto, 1980: 8, fig. 11.

Descrição: lórica hialina grande e alongada. Colar cônico truncado invertido pouco a bastante desenvolvido; borda oral lisa. Algumas lóricas apresentam colar expandido lateralmente, de lados curvados para fora como na Fig. 89. Lados paralelos no quarto anterior, tornando-se discretamente divergentes e convexos até o quarto posterior, quando formam uma pequena constrição que delimita a região aboral. Esta apresenta parede expandida e contém as cristas aborais. Cristas quase retas em número de 4-6, iniciando-se numa extremidade aboral semelhante um pequeno cone (nem sempre visível). Os lados da lórica podem apresentar-se algo irregulares em toda sua extensão.

Medidas (8 ind.) comp. 255-351 $\mu$; larg. 14-15 $\mu \mathrm{m}$; D.O. 21-25 $\mu \mathrm{m}$.

Comentários: espécie razoavelmente polimórfica. BALECH (1959) e BALECH \& Souto (1980) incluem como sinônimas as espécies: S. attenuata Jörgensen, $S$. gracilis Kofoid \& Campbell e S. sinistra Kofoid \& Campbell, considerando que apenas as dimensões do D.O. associada ao desenvolvimento da borda oral não seriam critérios suficientes para separar as espécies. $S$. gracilis apresenta colar desenvolvido, sendo muito semelhante à lórica ilustrada na Fig. 89. Para discussão de espécies sinônimas, veja BALECH (1959).

Ocorrência nas amostras: Operação Monitor IV (estações 8/100 m, 9/10 m, 9/100 m); Operação Sueste IV (estações 6612, 6614); Projeto COROAS (estações 1, 9, 10, 13/150-100 m, 17/ 130-100 m).

\section{Salpingella laminata Kofoid \& Campbell, 1939}

Fig. 54

Salpingella laminata Kofoid \& Campbell, 1939: 388, pl. 33, fig. 1.

Descrição: lórica hialina curta, em forma de projétil. Colar discreto em forma de cone truncado invertido ligeiramente expandido. O corpo apresenta diâmetro menor na base do colar (constricção) e seus lados são côncavos até a região aboral. Esta se apresenta cônica de extremidae subaguda. Não há cristas aborais. Parede bastante delicada, quase transparente.

Medidas (2 ind.) comp. 60-64 $\mu \mathrm{m}$; larg. da constrição $13 \mu \mathrm{m}$; D.O. 16,6 $\mu \mathrm{m}$.

Comentários: espécie com dimensões semelhantes a Salpingella aff. laminata Kofoid \& Campbell (1939) (comp. 73 $\mu \mathrm{m}$, D.O. $15 \mu \mathrm{m}$, constrição $12 \mu \mathrm{m}$ ) mas sem cristas aborais, e com região aboral menos desenvolvida.

Ocorrência nas amostras: Operação Sueste IV (estações $6599,6604)$.

\section{Salpingella subconica Kofoid \& Campbell, 1929}

\section{Fig. 55}

Salpingella subconica Kofoid \& Campbell, 1929: 355, fig. 676.Balech, 1959: 53, pl. 20, figs 293-294.- Balech, 1971: 183, pl. 38, figs 749-750.

Descrição: lórica hialina alongada e afilada, apresentando colar discreto em forma de cone truncado invertido ligeiramente expandido. O corpo apresenta diâmetro menor na base do colar e lados paralelos até a parte mediana, quando se dilata e os lados tornam-se convexos. Na região aboral, os lados voltam a convergir. Cristas aborais em número de seis, partindo da extremidade aboral e atingindo aproximadamente $1 / 3$ do comprimento total. Parede bastante delicada, quase transparente.

Medidas ( 2 ind.) comp. 146-148 $\mu \mathrm{m}$; larg. mediana $12 \mu \mathrm{m}$; D.O. $17 \mu \mathrm{m} ; 6$ cristas aborais; comp. das cristas 30-31 $\mu \mathrm{m}$.

Comentários: não foi observado cilindro aboral terminal, como em BALECH (1959).

Ocorrência nas amostras: Operação Sueste IV (estações 6599, 6600, 6601, 6603, 6604); PROANTAR XI (estação 11, 12).

\section{Steenstrupiella gracilis (Jörgensen, 1924)}

Fig. 56

Amphorella gracilis Jörgensen, 1924: 21, fig. 19.

Steenstrupiella gracilis; Kofoid \& Campbell, 1929: 313, fig. 597.-

Hada, 1938: 167, Fig. 8.- Campbell, 1942: 114.

Descrição: lórica hialina; região oral composta por um colar cônico truncado invertido de lados ligeiramente convexos. Abaixo do colar inicia-se o cesto cilíndrico, de lados paralelos até a região aboral, quando se tornam convergentes para formar uma extremidade aboral obtusa. Parede lisa, sem qualquer ornamentação (cristas ou estrias). O colar apresenta parede espessada, a qual se inicia na borda oral e termina na base do colar. Borda oral lisa. 
Medidas (14 ind.) comp. 62-68 $\mu \mathrm{m}$; D.O. $32 \mu \mathrm{m}$.

Comentários: Souto (1981) e CAMpBELl (1942) registram 4-6 estrias muito delgadas na região aboral, não encontradas nas lóricas de nosso material.

Ocorrência nas amostras: Operação Sueste IV (estações 6612, 6615, 6616); Projeto COROAS (estações 1, 3, 9, 10, 13/ 150-100 m, 17/130-100 m); PROANTAR XI (estações 3-5).

\section{Steenstrupiella steenstrupii (Claparéde \& Laachmann, 1858)} Figs 57-59

Tintinnus steenstrupii Claparéde \& Laachmann, 1858: 200, pl. 8 , fig. 5.

Steenstrupiella steenstrupii; Kofoid \& Campbell, 1929: 314, fig. 596.- Balech, 1959: 54, pl. 20, figs 299-302.- Balech, 1962: 107, pl. 13, figs 167-169.- Souto, 1970b: 222, fig. 8.

Descrição: lórica hialina; região oral cônica truncada invertida de lados retos. Abaixo do colar os lados são paralelos ou discretamente convergentes até a região aboral, quando se tornam divergentes, formando uma dilatação. Extremidade aboral obtusa a subaguda. Cristas aborais longitudinais paralelas, estendendo-se ou não por toda a região aboral. Às vezes não são visualizadas (inexistentes?). Certas lóricas apresentam um espessamento da parede, que se inicia na borda oral e termina na dilatação aboral, sendo mais pronunciado no colar, formando uma figura semelhante à letra "L" invertida. Borda oral lisa.

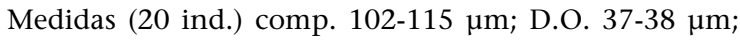
larg. na região mediana $18 \mu \mathrm{m}$; larg. na dilatação da região aboral 17-18 $\mu \mathrm{m}$; larg. antes do espessamento $15 \mu \mathrm{m}$; altura do colar 9-10 $\mu \mathrm{m}$; espessura da parede abaixo da extremidade oral $3 \mu \mathrm{m}$.

Comentários: espécie semelhante a $S$. pozzii Balech, porém esta apresenta D.O. maior $(42-49 \mu \mathrm{m})$ e cristas mais desenvolvidas, em número de seis (BALECH 1971). HADA (1938) encontrou lóricas com e sem cristas aborais. No presente trabalho, as lóricas encontradas apresentaram morfologia muito parecida às ilustradas em BALECH (1971) e Souto (1981) para S. pozzii, podendo causar confusão na identificação. Portanto, o critério para separar as duas espécies foi o diâmetro oral. As espécies $S$. robusta Kofoid \& Campbell e S. steenstrupii var. robusta (Kofoid \& Campbell) Balech foram consideradas sinônimas.

Ocorrência nas amostras: Operação Monitor IV (estação 10/100 m); Operação Sueste IV (estações 6602-6604); Projeto COROAS (estações 1, 3, 9, 10); PROANTAR XI (estações 1, 4-10, 15).

\section{Xystonellidae Kofoid \& Campbell, 1929 Parundella caudata (Ostenfeld, 1899) Figs 90-91}

Tintinnus caudatus Ostenfeld, 1899: 63.

Parundella caudata; Kofoid \& Campbell, 1929: 228, fig. 442.Balech \& Souto, 1981: 46, pl. 2, figs 1-2.

Descrição: lórica hialina caliciforme alongada. Corpo cilíndrico até a região mediana (em algumas lóricas os lados são discretamente convergentes), tornando-se cônico de lados convexos na metade posterior. Apêndice caudal cônico pequeno, provido de cristas longitudinais que atingem a base do corpo. Dardo cônico pontiagudo. Borda oral lisa. Parede trilaminar, alvéolos da parede secundária pouco evidentes.

Medidas (13 ind.) comp. 106-142,2 $\mu \mathrm{m}$, D.O. $28 \mu \mathrm{m}$.

Ocorrência nas amostras: Operação Monitor IV (estações 6/100 m, 8/10 m, 12/100 m); Operação Sueste IV (estações 6599, 6600); Projeto COROAS (estações 1, 3, 9, 13/150-100 m, 17/ 130-100 m).

\section{Parundella pellucida (Jörgensen, 1924)} Fig. 92

Undella caudata Jörgensen, 1924: 46 (partim).

Parundella pellucida; Kofoid \& Campbell, 1929: 233, fig. 438.Balech, 1971: 177, pl. 39, fig. 752.- Balech \& Souto, 1981: 46, pl. 2, figs 6-7; pl. 3, figs 1-2.

Descrição: lórica hialina caliciforme e alongada, composta por corpo e cauda com dardo aboral. Corpo de parede côncava (lados convergentes) até a porção mediana, tornando-se dilatada na metade posterior, de lados convexos, onde localiza-se a maior parte do protozoário. Em seguida ocorre uma cauda cônica afilada, terminando em dardo agudo. Parede trilaminar, a secundária composta por pequenos alvéolos (v. fig. 92 na cauda), dispostos em estrias curvadas. Na região oral a parede é delgada. Protozoário ocupa 60-70\% da lórica; três macronúcleos.

Medidas (3 ind.) comp. 140-158,5 $\mu \mathrm{m}$, D.O. 40-41 $\mu \mathrm{m}$, comp. do dardo $18 \mu \mathrm{m}$.

Comentários: diferencia-se de $P$. caudata pela presença de dardo e parede sinuosa. A taxonomia das espécies próximas foi esclarecida por BALECH \& Souto (1981).

Ocorrência nas amostras: Operação Monitor IV (estação 10/100 m); Operação Sueste IV (estações 6599-6602, 6604); Projeto COROAS (estações 3, 9, 13/150-100 m, 17/130-100 m).

\section{Xystonella longicauda (Brandt, 1906)}

Fig. 60

Cyttarocylis acus var. b longicauda Brandt, 1906, pl. 50, figs 1-4.Brandt, 1907: 26.

Xystonella longicauda; Kofoid \& Campbell, 1929: 237, fig. 451.Souto, 1970b: 201, fig. 43.

Descrição: lórica hialina caliciforme, cônica e alongada. Borda oral lisa, circundada por espessamento oral triangular, freqüentemente pouco desenvolvido ou inexistente. O cesto apresenta lados convergentes. Em algumas lóricas pode apresentar-se inflado no terço posterior. Extremidade aboral cônica afilada de ápice agudo. Parede composta por uma única camada de alvéolos poligonais bastante tênues, exceto no espessamento oral, onde há três camadas mais evidentes.

Medidas (6 ind.) comp. 230-261 $\mu \mathrm{m}$; larg. 57-59 $\mu \mathrm{m}$; D.O. 38-43 $\mu \mathrm{m}$.

Revista Brasileira de Zoologia 21 (3): 605-628, setembro 2004 
Ocorrência nas amostras: Operação Sueste IV (estações 6600-6604, 6616-6619); PROANTAR XI (estações 1-3, 6, 15); Projeto COROAS (estações 1, 7, 9, 13/150-100 m; 5/50-0 m).

\section{Xystonella treforti (Daday, 1887) Laackmann, 1910} Fig. 61

Cyttarocylis treforti Daday, 1887: 515/579, pl. 21, fig. 1. Xystonella treforti; Laackmann, 1910: 449.- Balech, 1959: 42, pl. 15, fig. 220.

Descrição: lórica hialina e cilíndrica até aproximadamente metade de seu comprimento, quando torna-se cônica, formando um apêndice caudal. Extremidade aboral provida de espessamento triangular, suportando um dardo cônico de ápice agudo. Borda oral circundada por duas projeções (ou rebordas) da parede (interna e externa) separadas entre si por uma canaleta. Projeção interna lisa, e externa com dentículos diminutos. Parede espessada, provida de alvéolos grandes hexagonais, exceto próximo à extremidade oral, onde são pequenos e dispostos em 4-6 fileiras. No apêndice caudal também ocorrem alvéolos pequenos, que se tornam pontuações no espessamento aboral. Alguns indivíduos apresentam a metade anterior de lados ligeiramente convergentes, conferindo aparência cônica à lórica.

Medidas (28 ind.) comp. 380-420 $\mu \mathrm{m}$; larg. máxima 73 $\mu \mathrm{m}$; D.O. $58 \mu \mathrm{m}$; larg. da extremidade oral $80 \mu \mathrm{m}$; comp. do apêndice caudal 125-131 $\mu \mathrm{m}$; comp. dardo $32 \mu \mathrm{m}$; 3-4 alvéolos em $10 \mu \mathrm{m}$.

Ocorrência nas amostras: Operação Sueste IV (estações 66599, 6602-6604, 6613, 6616); Projeto COROAS (estações 1, 3, 5, 9, 10, 13/150-100 m, 17/130-100 m; 19/130-70 m); PROANTAR XI (estações 1-3, 5-12, 15).

\section{Xystonellopsis cymatica (Brandt, 1906) Figs 62-63, 93}

Cyttarocylis cymatica Brandt, 1906: 6, 25, pl. 44, figs 3-4. Xystonellopsis cymatica; Jörgensen, 1924: 50-52, figs 57a-57b.Hada, 1938: 150, fig. 66.- Balech, 1968: 179, pl. 3, Figs 2023.

Descrição: lórica hialina caliciforme. Região oral cilíndrica ou subcilíndrica até a metade anterior, tornando-se cônica de lados discretamente convexos. Em seguida, forma-se um pedúnculo mais estreito, sub-quadrangular com base dilatada, terminando em um dardo cônico pontiagudo. A base do pedículo apresenta vértices agudos, ás vezes modificados em espinhos. Parede bilaminar, envolvendo alvéolos prismáticos grandes, exceto na margem oral, onde são menores. Tipicamente ocorre um anel suboral de parede espessada para o exterior; sua parte anterior é mais abrupta do que a posterior, a qual apresenta lados que se continuam mais suavemente coma parede da lórica. Uma pequena franja oral é limitada a partir do anel.
Medidas (3 ind.) comp. 238-240 $\mu \mathrm{m}$; D.O. $44 \mu \mathrm{m}$; comp. dardo $30 \mu \mathrm{m}$.

Comentários: dimensões coincidentes com as de BALECH (1968). Dimensões do pedículo maiores do que em HaDA (1938) e BALECH (1968). Concordamos com este autor, que incluiu $X$. cyclas Kofoid \& Campbell (antes X. cymatica var. $b$ Brandt) e $X$. spicata (Brandt) Jörgensen nas sinonímias de X. cymatica, por considerar que a presença de estrias e pedículo evidenciado não seriam caracteres suficientes para separar as espécies.

Ocorrência nas amostras: Operação Sueste IV (estações 6602, 6604, 6615); Projeto COROAS (estações 1, 7, 9, 13/150$100 \mathrm{~m}, 17 / 130-100 \mathrm{~m} ; 19 / 130-70 \mathrm{~m}$ ).

\section{Xystonellopsis dahli (Brandt, 1906) Figs 64, 94}

Undella heros var. $b$ dahli Brandt, 1906, pl. 43, figs 1-3.- Brandt, 1907: 24.

Xystonellopsis dahli; Kofoid \& Campbell, 1929: 245, fig. 483.-

Balech, 1962: 97, pl. 12, fig. 144.

Descrição: lórica hialina subcilíndrica a cilíndrica, de lados paralelos até pouco mais da metade de seu comprimento, tornando-se fortemente convergentes no terço posterior. Região aboral cônica truncada de lados retos, terminada em um apêndice caudal cônico e afilado de extremidade aguda. Parede bilaminar envolvendo uma camada de pequeníssimos prismas, sem ornamentações (lisa), exceto no apêndice caudal, provido de prismas pardo-amarelados e cristas longitudinais torcidas evidentes.

Medidas (37 ind.) comp. 364-430 $\mu \mathrm{m}$; D.O. 58-61 $\mu \mathrm{m}$.

Comentários: difere de X. kramerii (Brandt) Kofoid \& Campbell por apresentar dimensões menores e cristas longitudinais menos desenvolvidas. As medidas de X. kramerii são:

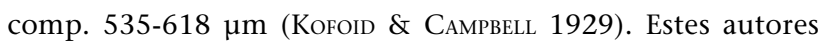
mencionam a existência de base ("skirt") do pedículo em $X$. kramerii como uma característica distintiva, mas em seu trabalho posterior (KoFoID \& CAMPBELL 1939) descrevem que há apenas vestígios da base. Portanto, se também considerarmos que o comprimento da lórica é um critério fraco para diferenciar espécies, $X$. dahli poderia ser considerada uma forma menor desta última. BALECH (1962) interpretou a terminação da cauda como um dardo irregular pouco delimitado, mas no presente trabalho, consideramos como parte do apêndice caudal.

Ocorrência nas amostras: Operação Sueste IV (estações 6659, 6602-6604, 6611-6616); Projeto COROAS (estações 1, 7, 9, 13/150-100 m, 17/130-100 m, 19/130-70 m).

\section{Xystonellopsis dicymatica (Brandt, 1906) Figs 65-66}

Cyttarocylis dicymatica Brandt, 1906, pl. 46, figs 1-2, pl. 68, fig. 10.- Brandt, 1907: 6, 25, 33.

Xystonellopsis dicymatica; Kofoid \& Campbell, 1929: 245, fig. 467. 


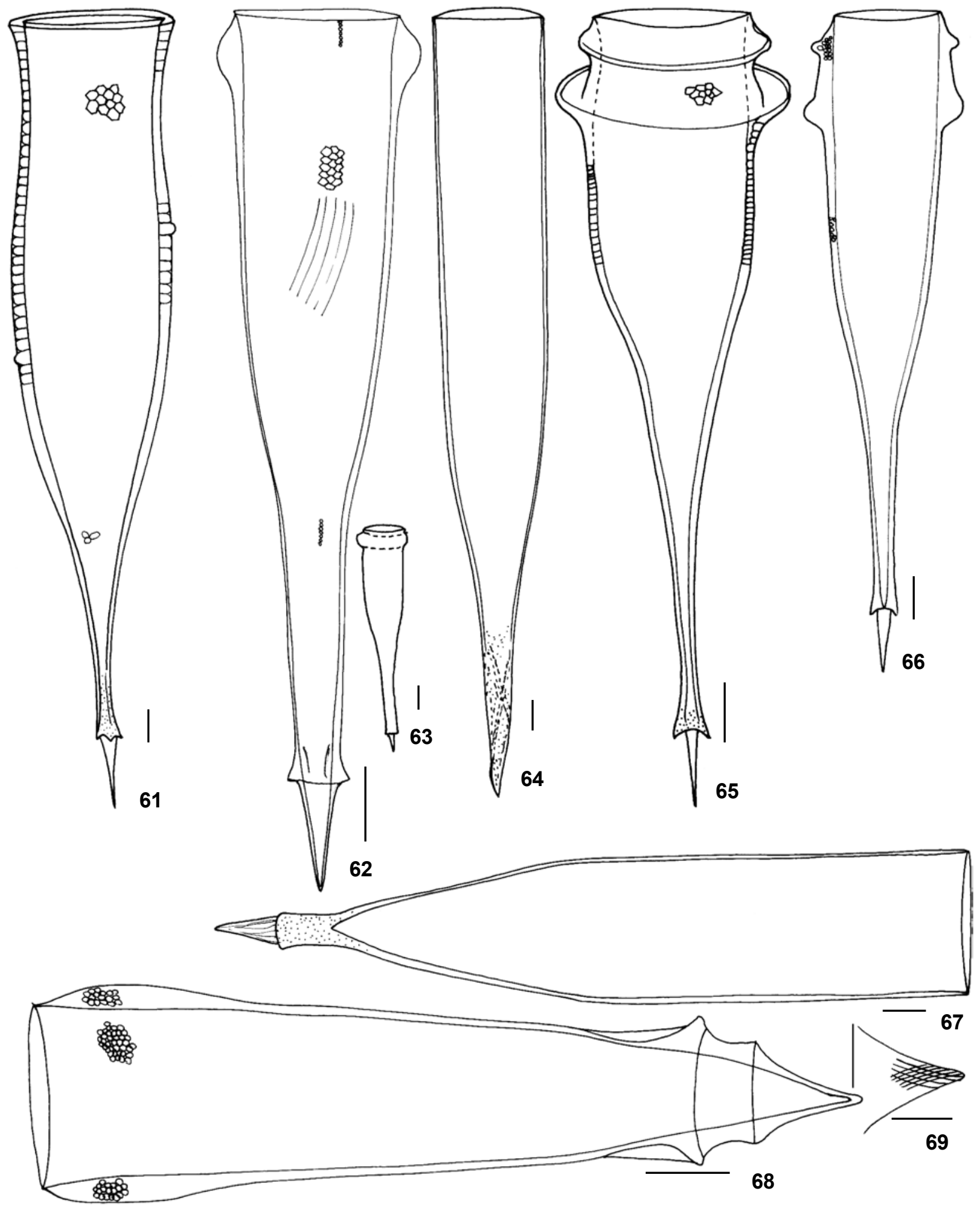

Figuras 61-69. (61) Xystonella treforti; $(62,63)$ Xystonellopsis cymatica; $(64)$ Xystonellopsis dahli; $(65,66)$ Xystonellopsis dicymatica; $(67)$ Xystonellopsis gaussi; $(68,69)$ Xystonellopsis paradoxa. Note as estrias decussadas em $(69)$. Barras de escala $=20 \mu \mathrm{m}$. 
Descrição: lórica hialina em forma de cálice alongado. Região oral lisa; região suboral provida de dois espessamentos anelares da parede, de formato triangular em corte transversal. Espessamentos de dimensões semelhantes ou, às vezes, desiguais, sendo o primeiro (mais próximo da região oral) menor do que o segundo. Aproximadamente na metade da extensão da lórica a parede torna-se estreita, voltando a dilatar-se na extremidade aboral, onde apresenta pequenas dilatações. Dardo afilado cônico, de ápice agudo. Parede composta por lamelas interna e externa (sensu KoFoID \& CAMPBELL 1939) envolvendo alvéolos hexagonais evidentes, que diminuem de tamanho próximo a extremidade oral e na região aboral.

Medidas (4 ind.) comp. 245-265 $\mu \mathrm{m}$; D.O. $49 \mu \mathrm{m}$; comp. dardo 26-28 $\mu \mathrm{m}$.

Ocorrência nas amostras: Operação Monitor IV (estação 9/100 m, 10/100 m); Operação Sueste IV (estações 6604, 6614); Projeto COROAS (estações 1, 9, 13/150-100 m).

\section{Xystonellopsis gaussi (Laackmann, 1910) Figs $67,95-98$}

Undella heros var. gaussi Laackmann, 1910: 429, 468, 476-478, 493, pl. 49, figs 23-24.

Xystonellopsis gaussi; Kofoid \& Campbell, 1929: 246, fig. 480.Sousa e Silva, 1954: 219, pl. 5, figs 7-9.- Souto 1970b: 201, fig. 44.

Descrição: lórica hialina de lados paralelos a ligeiramente convergentes até pelo menos a metade de seu comprimento, tornando-se fortemente convergentes no terço posterior. Região aboral cônica truncada terminada em um espessamento quadrangular de base alargada (pedículo, segundo a terminologia de Sousa \& SiLva 1954), suportando um dardo cônico e afilado de extremidade aguda. Parede bilaminar e sem ornamentações (lisa), envolvendo uma camada de pequeníssimos prismas (visíveis em 1000x), exceto na região aboral, onde é provida de prismas pardo-amarelados e 5-8 cristas longitudinais retas a torcidas, evidentes. Algumas diatomáceas penadas aderidas ás lóricas foram encontradas com freqüência.

Medidas (25 ind.) comp. 390-494 $\mu \mathrm{m}$; larg. 73-80 $\mu \mathrm{m}$; D.O. 65-72 $\mu \mathrm{m}$; dardo 36-43 $\mu \mathrm{m}$. A maioria das lóricas apresentou comprimento superior a $440 \mu \mathrm{m}$ e D.O. maior do que $71 \mu \mathrm{m}$.

Comentários: algumas lóricas apresentavam diatomáceas penadas na superfície externa, como mostrado nas Figs 79 e 85. BaLech (1962) e Sousa e Silva (1954) mencionam a presença de prismas diminutos compondo a parede. Diferencia-se de $X$. heros (Cleve) Kofoid \& Campbell por apresentar pedículo de base menos dilatada, paredes retas (ou quase) até pelo menos o terço anterior da lórica. As medidas se enquadram bem nas dos exemplares de Souto (1970b, como X. heros). A taxonomia da espécie é confusa, pois diferentes autores ilustram lóricas sob o nome $X$. heros que corresponderiam, na realidade, à X. gaussi. Por exemplo, as figuras de HADA (1938, fig.68) e SouTo (1970b, fig.44) corresponderiam à $X$. gaussi, se levarmos em consideração o critério original de Cleve (in BALECH 1962), que diferencia as duas espécies: lóricas cônicas em $X$. heros e cilíndricas em $X$. gaussi. Já em BaLeCH (1962) a ilustração da fig.143 (Pl. 12) para X. heros estaria correta. Balech e Hada argumentaram ainda que $X$. gaussi seria sinônimo de $X$. heros. Em Kofoid \& Campbell (1929) a figura 484 reproduz a original de Cleve para $X$. heros (descrita sob Undella heros), e então se nota bem a lórica cônica e o pedículo mais dilatado. Inexplicavelmente, porém, os mesmos autores colocaram como sinônima a fig.1, pl.42 de BRANDT (1907), que se enquadraria muito bem em $X$. gaussi! Portanto, se o critério morfologia da lórica discutido acima for considerado diagnóstico, as duas espécies seriam válidas, procedimento este adotado no presente trabalho. Então, se corretas, as figuras de Souto (1970b, 1981) seriam ilustrações satisfatórias da espécie $X$. heros (e não de $X$. gaussi como determinado pela autora!) e citação nova para o Atlântico Sul Ocidental. As lóricas encontradas no presente trabalho encaixaram-se bem em $X$. gaussi. Para finalizar, as medidas e as ilustrações de LAACKMANn (1910: 477, Tf. 49, figs 23,24$)$ para sua nova variedade Undella heros var. gaussi, mais tarde elevada a categoria de espécie por KofoId \& CAMPBEL (1929) como X. gaussi, mostram perfeitamente uma lórica cilíndrica e provida de espessamento na base do pedículo. Na Fig. 96a lórica apresenta lados paralelos apenas em seu quarto anterior, as cristas são mais robustas e o dardo mais dilatado em sua base, características que a posicionam como uma forma de transição entre $X$. gaussi e $X$. heros.

Ocorrência nas amostras: Operação Sueste IV (estações 6599-6600, 6602-6604, 6606, 6611, 6616-6618); Projeto COROAS (estações 1, 7, 9, 13/150-100 m 17/130-100 m; 19, 130$70 \mathrm{~m})$.

\section{Xystonellopsis paradoxa (Cleve, 1900) Figs 68-69, 99}

Undella paradoxa Cleve, 1900: 974-975, fig. 12.

Xystonellopsis paradoxa; Jörgensen, 1924: 51-52.- Kofoid \& Campbell, 1929: 249, fig. 463.- Balech, 1962: 96, pl. 11, fig. 137.

Descrição: lórica hialina cônica, terminando em um dardo alargado de extremidade aguda. Parede com vários espessamentos que se projetam externamente. A região suboral apresenta espessamento abrangendo $1 / 4$ da lórica, estreitando-se novamente até a região aboral. Nesta ocorre novo espessamento, composto por duas projeções, onde a primeira e maior é projetada para o exterior, formando um véu (ou anel) conspícuo. A segunda projeção é menos evidente e suporta o dardo cônico. Parede constituída por camadas de pequenos alvéolos em toda a extensão da lórica. Estrias delicadas partem da projeção maior até a extremidade do dardo, dispostas em linhas curvas e cruzando-se entre si (Fig. 69).

Medidas (12 ind.) comp. 184-201 $\mu \mathrm{m}$; larg. 48-49 $\mu \mathrm{m}$; D.O. $43 \mu \mathrm{m}$; comp. do dardo 25-29 $\mu \mathrm{m}$; 9-10 retículos em $10 \mu \mathrm{m}$.

Ocorrência nas amostras: Operação Monitor IV (estação 1/10 m, 2, 4, 8/10 m, 10/100 m); Operação Sueste IV (estações 6602-6605, 6612, 6614, 6616-6618); Projeto COROAS (estações $1,3,7,9,13 / 150-100 \mathrm{~m})$. 


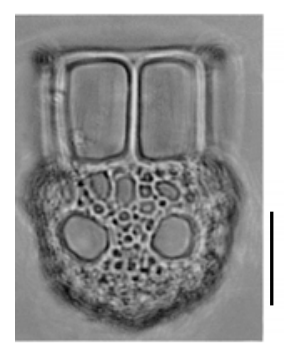

70

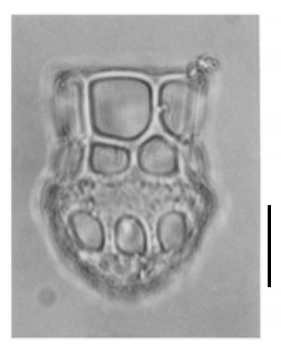

75

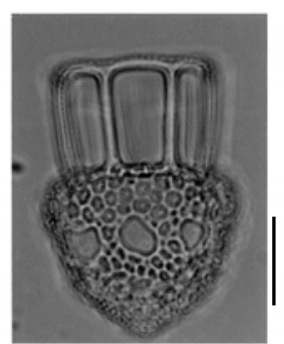

71

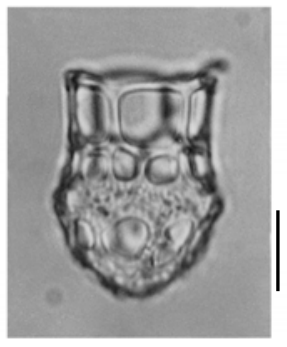

76

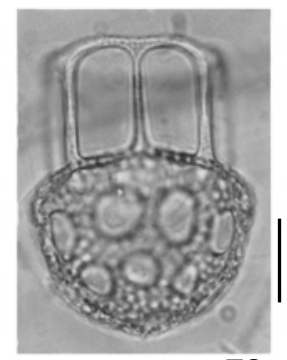

72

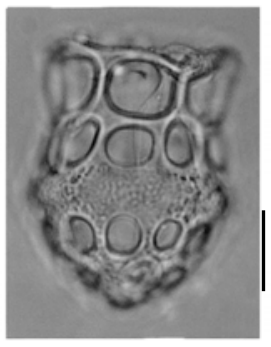

77

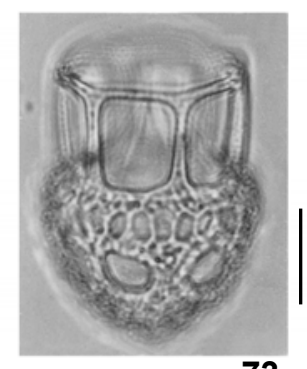

73

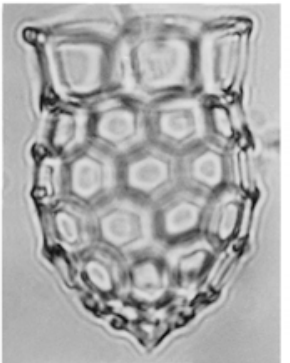

78

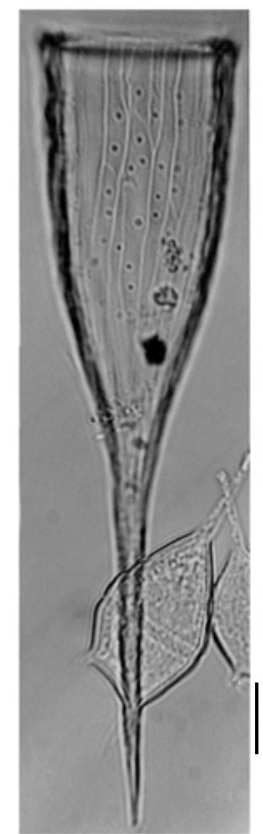

81

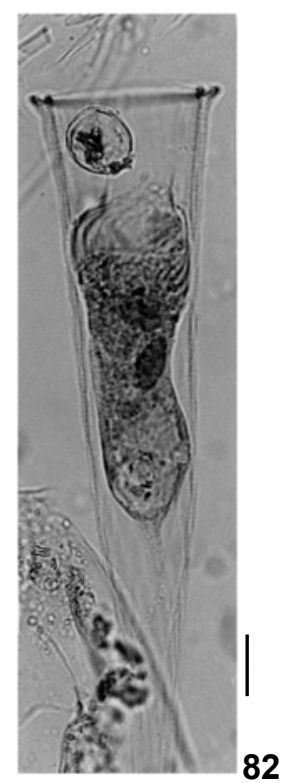

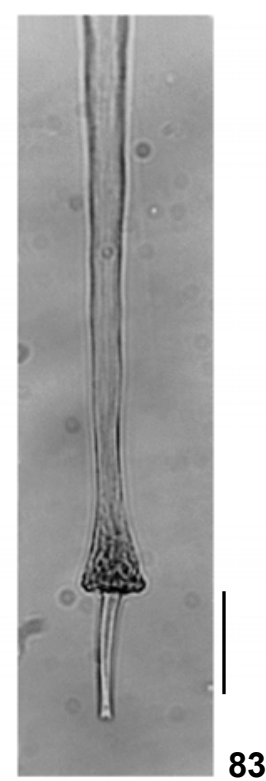

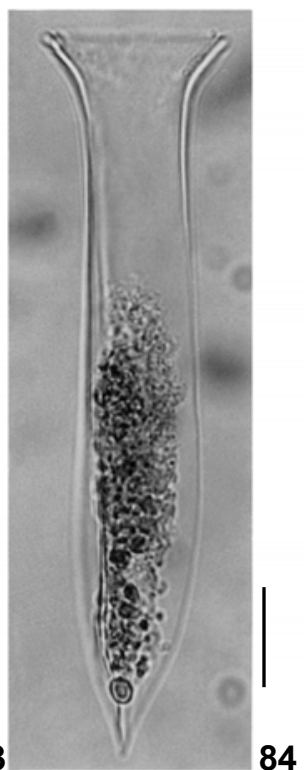

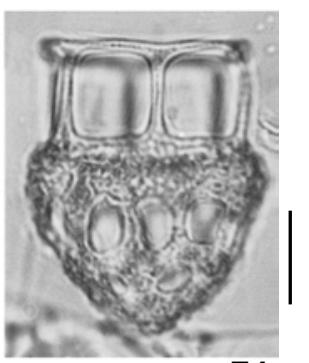

74

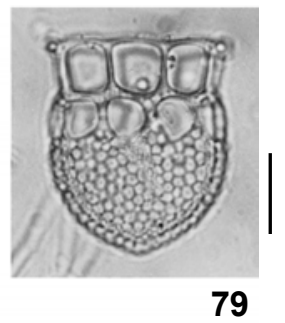

79

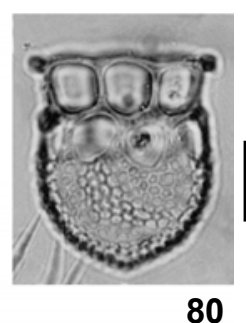

80

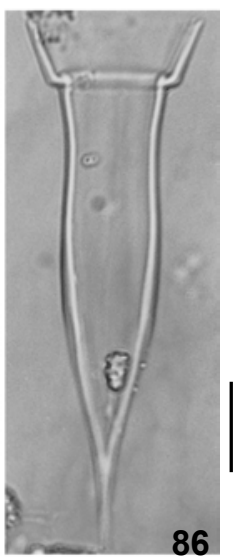

Figuras 70-86. $(70,74)$ Dictyocysta elegans var. lepida; $(75,77)$ Dictyocysta elegans var. speciosa; $(78)$ Dictyocysta mitra; $(79,80)$ Dictyocysta mitra (?); (81) Rhabdonella elegans; $(82,83)$ Rhabdonellopsis apophysata, em (82) note o zoóide e pedúnculo posterior; (84) Amphorellopsis acuta; (85) Eutintinnus medius; (86) Ormosella bresslaui. Barras de escala $=20 \mu \mathrm{m}$.

\section{DISCUSSÃO}

A maioria dos tintininos determinados nas amostras dos diferentes cruzeiros oceanográficos realizados na região Sueste-Sul do Brasil é característica de águas subtropicais e tropicais, ocorrendo em diferentes oceanos do mundo (LAACKMANN
1907, 1910, Kofoid \& Campbell 1929, 1939, Hada 1938, 1970, Balech 1962, Souto 1981, Pierce \& Turner 1993). A riqueza em espécies foi elevada (87 táxons infragenéricos), particularmente quando comparada às associações de águas mais frias das regiões subantártica e antártica, onde seu número oscila entre 


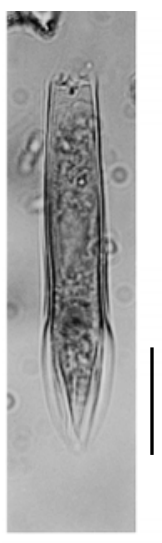

87

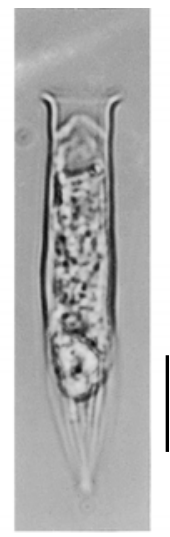

88

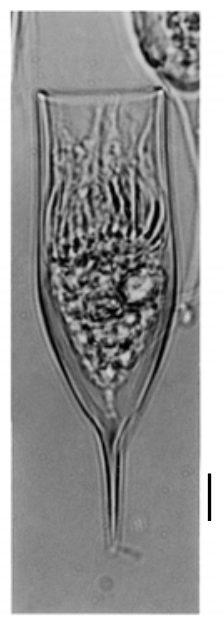

90

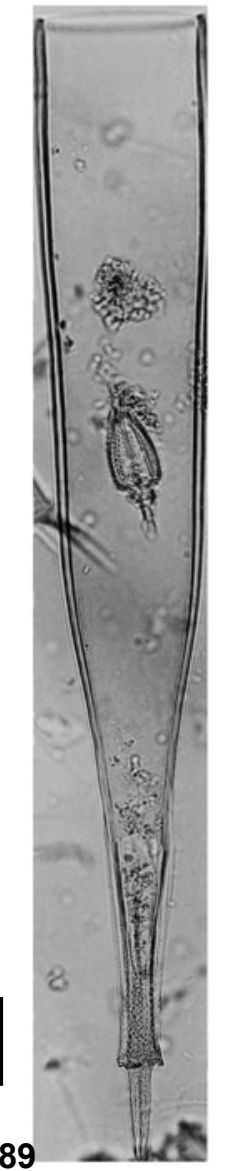

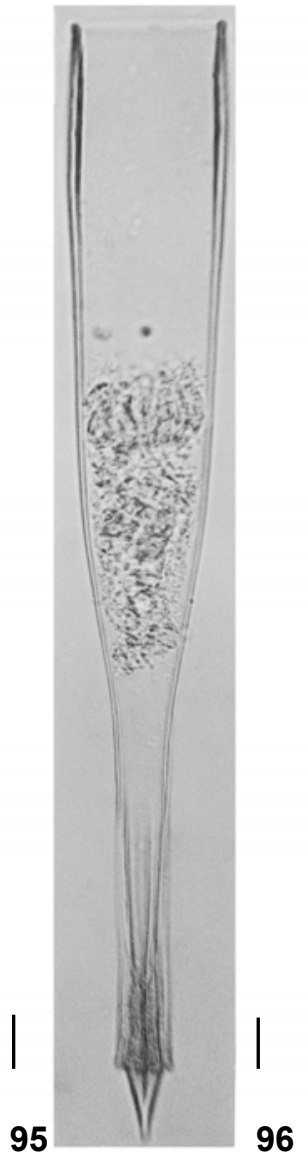
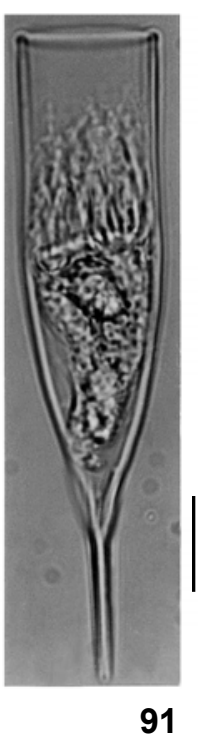

91

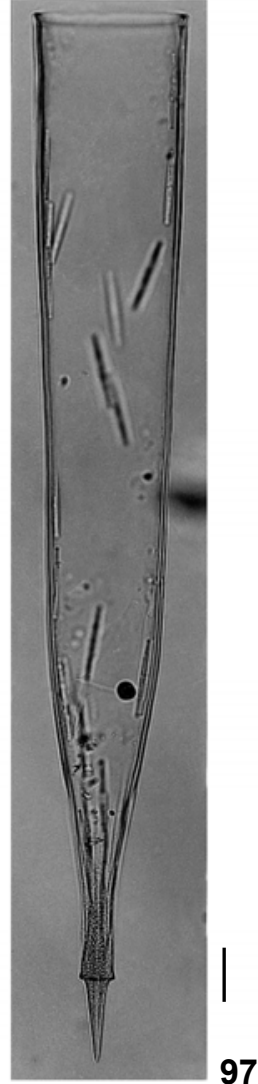

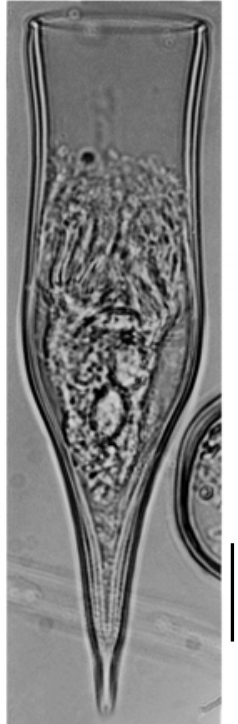

92

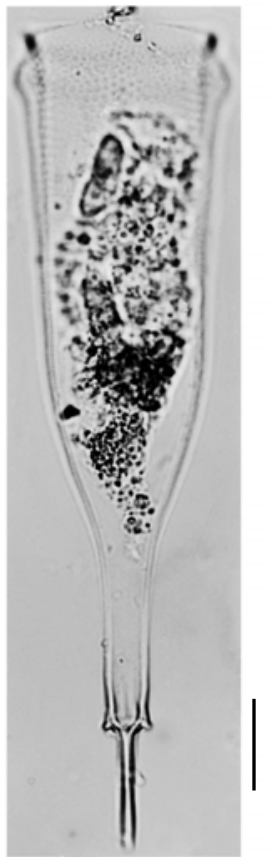

93
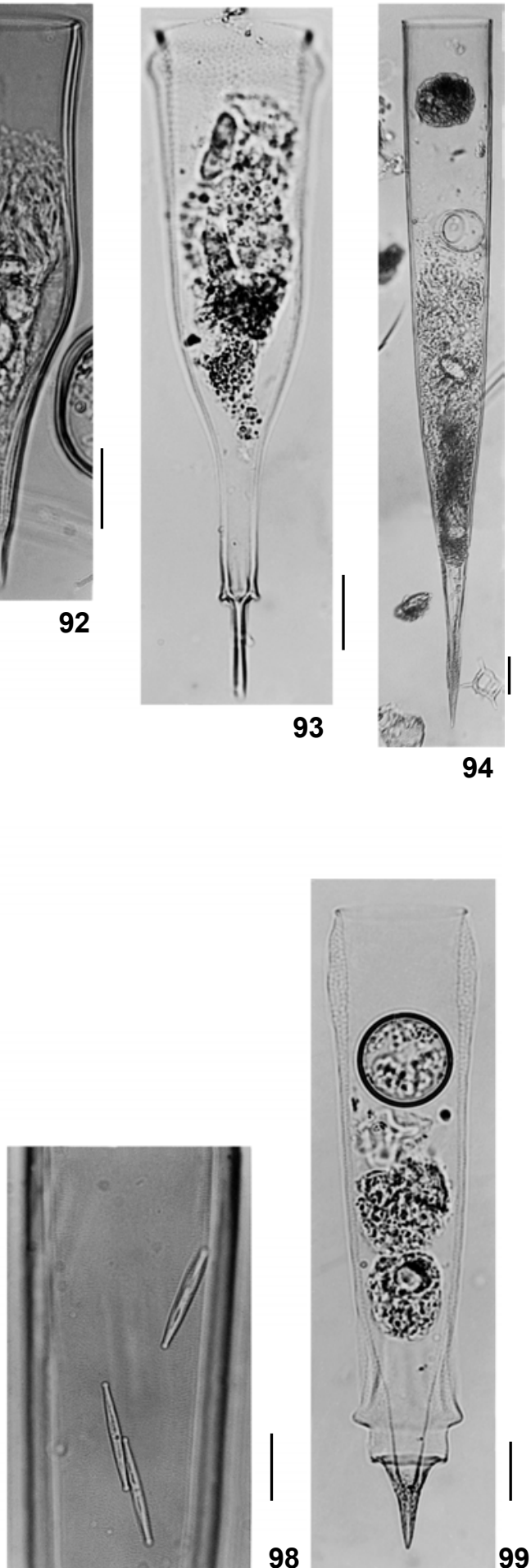

Figuras 87-99. (87) Salpingacantha unguiculata (?); (88) Salpingella curta; (89) Salpingella glockentoegerii; (90, 91) Parundella caudata caudata; (92) Parundella pellucida; (93) Xystonellopsis cymatica; (94) Xystonellopsis dahli; $(95,98)$ Xystonellopsis gaussi, em (98) detalhe do cesto, mostrando diatomáceas penadas aderidas à parede da lórica; (99) Xystonellopsis paradoxa. Barras de escala = $20 \mu \mathrm{m}$.

Revista Brasileira de Zoologia 21 (3): 605-628, setembro 2004 
12-30 espécies, embora suas densidades tendam a ser maiores (Hada 1970, Boltovskoy et al. 1989, GarRison \& Gowing 1993, FERNANDES 1999). Algumas espécies comuns e até abundantes em nosso material, como Climacocylis scalaria, Eutintinnus spp., Salpingacantha spp., Salpingella spp. e Steenstrupiella gracilis, surpreendentemente ainda não tinham sido registradas para a região. Suas lóricas são bastante delicadas e quase transparentes, dificultando sua localização durante a análise das amostras. Diferenças importantes também foram observadas quanto à composição das espécies determinadas nos diferentes cruzeiros oceanográficos. Nas estações referentes à Operação Sueste IV e Projeto COROAS as amostras foram muito mais ricas em espécies do que naquelas da Operação Monitor IV e PROANTAR XI, certamente devido ao maior volume de água filtrada pelas redes de plâncton. Entretanto, Amphorides amphora, Climacocylis scalaria, Eutintinnus lususundae, E. medius, E. fraknoi e Steenstrupiella steenstrupii foram abundantes apenas nas amostras do PROANTAR XI (Fernandes 1999, Fernandes \& BRAndini 1999), sendo raros ou inexistentes nas amostras das outras expedições. Neste caso, o método da filtração reversa utilizado no PROANTAR XI para reter os protozoários (com rede de $10 \mu \mathrm{m}$ e filtrando um menor volume de água) deve ter sido mais eficiente em reter estas espécies e em preservar melhor a integridade das lóricas dos tintininos do que as grandes redes de plâncton utilizadas na Operação Sueste IV e Projeto COROAS. Estas apresentavam maior abertura das malhas, e estiveram sujeitas à maior atrito resultante do arrasto vertical na coluna de água, o que também danifica as lóricas mais frágeis.

As citações de 23 novas ocorrências para águas brasileiras foram baseadas nas revisões de Souto (1981), Pierce \& Turner (1993) е de Тномpson et al. (1999), e são: Acanthostomella minutissima Kofoid \& Campbell, Albatrossiella agassizi Kofoid \& Campbell, Amplectella tricollaria (Laackmann) Balech, Clymacocylis scalaria (Brandt) Jörgensen, Clymacocylis scalaroides Kofoid \& Campbell, Clymacocylis sipho (Brandt) Kofoid \& Campbell, Codonella elongata Kofoid \& Campbell, Codonella galea Haeckel, Codonella olla Kofoid \& Campbell, Codonellopsis ostenfeldi (Schmidt) Kofoid \& Campbell, Cyttarocylis brandti Kofoid \& Campbell, Cyttarocylis conica (Brandt) Kofoid \& Campbell, Epicancella nervosa (Cleve) Kofoid \& Campbell, Epiplocylis blanda Kofoid \& Campbell, Eutintinnus latus (Jörgensen) Kofoid \& Campbell, Ormosella breslaui Kofoid \& Campbell, Poroecus curtus Kofoid \& Campbell, Salpingacantha unguiculata (Brandt) Kofoid \& Campbell, Salpingella curta Kofoid \& Campbell, Salpingella decurtata Jörgensen, Undella hyalina Daday, Undella pistillum Kofoid \& Campbell e Xystonellopsis dahli (Brandt) Kofoid \& Campbell. A espécie Xystonellopsis gaussi (Laackmann) Kofoid \& Campbell foi citada anteriormente por Souto (1970b) sob o nome X. heros (Cleve) Kofoid \& Campbell, e sua taxonomia já foi discutida na seção Resultados. As espécies Clymacocylis scalaria, Cyttarocylis brandti, Dictyocysta mülleri e Undella hyalina foram encontradas anteriormente no Oceano Atlântico Sul Ocidental apenas por LAaCKMANn (1910) em cinco amostras (26/08-18/09/1903) coletadas próximo ao meridiano de Greenwich $\left(0^{\circ}-20^{\circ} \mathrm{S}\right.$ e $\left.0^{\circ}-20^{\circ} \mathrm{W}\right)$, durante a expedição do navio alemão Meteor/1901-1903. Portanto, todas as outras citações dos tintininos mencionados acima correspondem ao primeiro registro naquele oceano.

Embora as amostras tenham sido coletadas em diferentes períodos, foi possível separar as espécies encontradas em duas associações: nerítica e oceânica. As espécies de águas neríticas ocorreram nas estações coletadas durante a Operação Sueste IV com profundidades inferiores à $50 \mathrm{~m}$, aproximadamente, e foram dominadas por Tintinnopsis spp., ocorrendo também Codonellopsis spp., Favella ehrenbergii, Helicostomella subulata, Leprotintinnus nordqvistii e Stylicauda platensis. Estes tintininos desenvolvem-se melhor em áreas influenciadas pelas águas de drenagem continental, menos salinas e com elevada turbulência, mantendo os sedimentos em suspensão, o que também favorece as espécies aglutinantes de partículas na lórica (KRSNIC 1987, CoRDEIro et al. 1997). As associações oceânicas ocorreram desde a plataforma média, com profundidades acima de $100 \mathrm{~m}$, em direção à áreas mais profundas. A maioria das espécies determinadas no presente trabalho pode ser incluída neste grupo, e os tintininos mais comuns foram Amphorides amphora, Climacocylis scalaria, Codonella spp., Dadayella ganimedes, Dictyocysta elegans lepida, Eutintinnus lususundae, E. medius, Rhabdonellopsis apophysata, Xystonella treforti e Xystonellopiss gaussii. Os poucos trabalhos realizados na plataforma sul brasileira relatam as mesmas espécies como as mais representativas nas amostras (Souto 1970a, b, amostras qualitativas; FERNANDES 2004). Nesta área predominam as águas da Corrente do Brasil com temperaturas superiores à $20^{\circ} \mathrm{C}$ e salinidades acima de 36,0 (Emílsson 1961, MatsuUra 1986). A biomassa fitoplanctônica, estimada a partir das concentrações de clorofila-a e contagem de células, geralmente é reduzida, predominando o nanoplâncton (BRANDINI 1988, 1990). O mesmo acontece com o microplâncton, incluindo os tintininos, ocorrendo em baixas densidades ao longo do ano, exceto durante ressurgências esporádicas, como observado na plataforma paranaense e em Cabo Frio, Rio de janeiro (BRANDini et al. 1989, VALENTIN 1989). Mais ao sul de nossa área de amostragem $\left(34^{\circ} \mathrm{S}-38^{\circ} \mathrm{S}\right)$, Thompson et al. (1999) encontraram as seguintes espécies abundantes: Ascampbeliella spp., $C$. scalaroides marshallae, D. mitra, E. medius, $R$. spiralis, X. longicauda f. typica e $X$. trefortii. Estes autores amostraram na parte norte da área conhecida como Zona de Transição ou Zona de Confluência Brasil-Malvinas, afetada por águas subantárticas, e registraram, juntamente com as espécies tipicamente tropicais, tintininos de águas frias como A. quadrilineata, D. lepida e $S$. pozzii. No caso específico da Operação Monitor IV, planejada como parte de um projeto maior organizado para estudar a periodicidade das ressurgências em Cabo Frio (DHN 1989), não foi detectado qualquer tintinino indicador de águas profundas em estações de superfície. Ao contrário, as espécies umbrófilas determinadas ocorreram sempre em estações à $100 \mathrm{~m}$ de profundidade. F.M. Pellizari (1996, dados não publicados) analisou as 
amostras de fitoplâncton do mesmo cruzeiro e não detectou qualquer alteração na biomassa e composição da comunidade. Estas observações corroboram os resultados de oceanografia física obtidos pela DHN (1989), mostrando que a ressurgência não ocorreu na época das amostragens, e a Água Central do Atlântico Sul manteve-se em profundidades maiores do que $100 \mathrm{~m}$.

Boltovsкoy $(1986,1989)$ discutiu os problemas relacionados à utilização e confiabilidade de espécies do plâncton como indicadoras de massas de água, comentando que estas espécies devem apresentar os requisitos fidelidade à massa de água, abundância razoável e distribuição horizontal ampla. Sugerimos que as espécies citadas anteriormente se enquadram razoavelmente nestes critérios, podendo ser boas indicadoras da Água Subtropical, pelo menos no sul do Brasil.

Durante a Operação Sueste IV abundâncias elevadas de tintininos foram observadas nas estações oceânicas mais ao sul (6597 à 6604). J.R.B. MACENO-SILVA (1991, dados não publicados) analisou os dados físicos e químicos coletados na mesma operação e mostrou a ocorrência de mistura entre águas subtropicais e subantárticas (Zona de Transição de BoLtovsKoY 1999) na área coberta por aquelas estações, observando alterações na temperatura e salinidade e aumento das concentrações de nutrientes e clorofila-a, promovendo o maior desenvolvimento da comunidade planctônica. Nesta área foram encontrados, entre outros, alguns tintininos normalmente registrados em águas mais frias, como Eutintinnus tubulosus, Parundella caudata, P. pellucida, Salpingella subconica, Undella subacuta e $U$. subcaudata (Souto 1970a, b, BALech \& Souto 1980, 1981).

Nas estações amostradas durante a Operação Monitor IV foram obtidas amostras de diferentes profundidades, e no Projeto COROAS foram realizados arrastos verticais estratificados em diferentes faixas de profundidade até a superfície, o que representa um interesse adicional importante relativo à distribuição vertical dos tintininos. Algumas espécies estiveram praticamente restritas às profundidades de 50-100 m, e são: Amplectella tricollaria, Brandtiella palliata, Codonella elongata, Daturella stramonium, Dictyocysta elegans speciosa, Epicancella nervosa, Petalotricha ampulla, Xystonellopsis cymatica, X. dahli e X. gaussi. Destas espécies, somente $X$. dahli e $X$. gaussi foram abundantes nos estratos de profundidade em que ocorreram, o que poderia gerar dúvidas quanto à representatividade das amostras se pretendemos discutir a sua distribuição vertical. Entretanto, algumas informações a seguir podem aumentar a confiabilidade dos dados. Por exemplo, estes tintininos não apareceram nos arrastos mais superficiais e, além disso, estiveram acompanhados por dinoflagelados e diatomáceas reconhecidamente umbrófilos (BALECH 1962, 1972, 1989, SouRnia 1982), como Ceratium gravidum, C. incisum, C. paradoxides, C. ranipes, Gossleriella tropica, Histioneis spp., Ornithocercus splendidus, Planktoniella sol e Triposolenia bidentata. As espécies de tintininos mencionadas enquadram-se bem no grupo I (decididamente umbrófilas) da classificação de BALECH (1972) para os tintininos que apresentam alguma afinidade por águas subsuperficiais e, conseqüentemente, potenciais indicadores de ressurgências. Em adição à lista fornecida por Balech, sugerimos a inclusão de X. dahli, X. gaussi, (que ocorreram em amostras a 150-100 $\mathrm{m}$ de profundidade durante o Projeto COROAS) e Epicancella nervosa, aparecendo em 4 amostras a $100 \mathrm{~m}$ de profundidade na Operação Monitor IV. Xystonella longicauda, X. treforti e Xystonellopsis paradoxa ocorreram em diferentes profundidades na coluna de água, não aparentando qualquer preferência por águas profundas. KRISNIC (1988) também registrou as maiores abundâncias de $X$. longicauda e $X$. treforti nos estratos superficiais do Mar Adriático, enquanto que $X$. cymatica e $X$. paradoxa apresentaram preferência por profundidades entre 50 e $200 \mathrm{~m}$. Por outro lado, Kimor \& GolandSKY-Baras (1981) não encontraram qualquer estratificação vertical de tintininos no Golfo de Elat, Mar Vermelho. Algumas das espécies abundantes nesta área (e presentes também em nosso material) mostraram afinidade por águas subsuperficiais em outros oceanos, como Codonellopsis orthoceras, Petalotricha ampula, Undella claparedei e Xystonella treforti (BALECH 1972). Os picos de abundância destas espécies no Golfo de Elat ocorreram no inverno, durante os eventos de desestratificação da coluna de água. Apenas Epiplocylis undella apresentou máximos durante a estratificação no verão. Como se pode notar, os estudos de KIMOR \& GOLANDSKY-BARAS (1981) foram realizados em uma região onde os fatores ambientais físicos que afetam a comunidade planctônica são distintos daqueles de águas oceânicas subtropicais/tropicais brasileiras. Nestas, uma termoclina sazonal permanente impede a desestratificação da coluna de água, gerando condições mais estáveis ao longo da zona fótica com gradientes de temperatura e de nutrientes (BRANDIN 1990) que podem conduzir ao isolamento e posterior adaptação das espécies a diferentes níveis de profundidade. Uma conseqüência deste processo seria, por exemplo, o aparecimento de comunidades planctônicas com afinidade por águas mais profundas, como tem sido observado para tintininos, dinoflagelados e algumas diatomáceas (BALECH 1972, 1989, SOURNIA 1982, VenRICK 1988).

\section{AGRADECIMENTOS}

À Diretoria de Hidrografia e Navegação e às tripulações dos NOc. "Almirante Saldanha" e "Barão de Teffé" (hoje "aposentados") pelo empenho em integrar biólogos e militares, e no auxílio durante as coletas. Ao Dr. Roberto Sassi (NEPREMAR, UFPB) e à MsC. Mayza Pompeu (IO, USP) pelas sugestões e correções do manuscrito. Aos professores MsC. João R. MacenoSilva (CEM, UFPR), Dr. Salvador A. Gaeta (IO, USP, coordenador do Projeto COROAS) e MsC. Mayza Pompeu (IO, USP), que gentilmente cederam as amostras de plâncton de rede e dados físicos e químicos referentes à Op. Sueste IV e ao Projeto COROAS. Financiado pelo Curso de Pós-graduação em Zoologia, UFPR, CNPq e Centro de Estudos do Mar, UFPR. Este trabalho é parte de Tese de Doutorado no curso de Pós-graduação em Ciências Biológicas, Zoologia, UFPR. O Dr. Olaf Mielke (Departamento de Zoologia, UFPR) forneceu valiosas orientações sobre nomenclatura zoológica a um botânico de formação. 


\section{REFERÊNCIAS BIBLIOGRÁFICAS}

Alder, V.A. 1999. Tintinnoinea, p. 321-384. In: D. Boltovskoy (Ed.). South Atlantic Zooplankton. Leiden, Backhuys Publishers, vol. 1, 868p.

BALECH, E. 1959. Tintinnoinea del Mediterraneo. Trabajos del Instituto Espanhol de Oeanografia, Madrid, 28: 1-88.

. 1962. Plancton de las campañas oceanograficas Drake I y II. Servicio de Hidrografia Naval, Buenos Aires, H.627: 1-57.

. 1968. Algunas especies nuevas o interessantes de tintinidos del Golfo de México y Caribe. Revista del Museo Argentino de Ciencias Naturales «Bernardino Rivadavia», Hidrobiología, Buenos Aires, 2 (5): 164-197.

—1971. Microplancton de la campaña oceanográfica Productividad III. Revista del Museo Argentino de Ciencias Naturales «Bernardino Rivadavia», Hidrobiología, Buenos Aires, 3 (1): 1-202.

1972. Los tintinidos indicadores de afloramiento de águas (Ciliata). Physis, Buenos Aires, 31 (83): 519-528.

. 1989. Los dinoflagelados del Atlantico Sudoccidental. Publicaciones del Instituto Espanhol de Oceanografia, Barcelona, (1): 1-310.

Balech, E. \& S. Souto. 1980. Los tintininos de la campaña oceanografica "Productividad IV". Parte I. Physis, Buenos Aires, 39: 1-8.

— 1981. Los tintininos de la campaña oceanografica "Productividad" IV. Parte II. Physis, Buenos Aires, 39: 41-49.

Boltovsкoy, D. 1986. Biogeography of the Southwestern Atlantic; overview, current problems and prospects, 14-24. In: A.C. Pierrot-Bulls (Ed.) Pelagic biogeography. Proc. of an International Conference, The Netherlands. UNESCO Technical Papers in Marine Sciences, Paris, 49: 1-300.

. 1989. Las zonas de transicion en la pelagial: biogeografia y paleobiogeografia, p. 9-24. In: F.P. BRANDINI (Ed.) Memórias do III Encontro Brasileiro de Plâncton. Curitiba, 100p.

. 1999. South Atlantic Zooplankton. Leiden, Backhuys Publishers, vol. 1, 868p.

Boltovskoy, D.; V.A. Alder \& F. Spinelli. 1989. Summer Weddel Sea microplankton: assemblage structure, distribution and abundance, with special emphasis on the Tintinnina. Polar Biology, Sttutgart, 9: 447-456.

BRANDINI, F.P. 1988. Composição e distribuição do fitoplâncton na região Sueste do Brasil e suas com as massas de água (Operação Sueste - julho/agosto 1982). Ciência e Cultura, São Paulo, 40 (4): 334-341.

.1990. Hydrography and characteristics of the phytoplankton in shelf and oceanic waters off southeastern Brazil during winter (july/august-1982) and summer (February/ March-1984). Hydrobiologia, Dordrecht, 196: 111-148.

Brandini, F.P.; C.L. B. Moraes \& C.A.C. Thamm. 1989. Shelf break upwelling, subsurface maxima of chlorophyll and nitrite, and vertical distribution of a subtropical nano- and microplankton community off southeastern Brazil (november 1985), p. 47-55. In: F.P. BRANDINI (Ed.). Memórias do III Encontro Brasileiro de plâncton. Curitiba, 100p.

Brandt, K. 1906. Die Tintinnodeen der Plankton-Expedition. Tafelerklärungen kuerzer Diagnose der neuen Arten. Ergebnisse der Atlantik Plankton-Expedition, Bremen, 3 (L.a.), 33p.

. 1907. Die Tintinnodeen der Plankton-Expedition. Systematischer Teil. Ergebnisse der Atlantik PlanktonExpedition, Bremen, 3 (L.a.), I-499p.

CAmpBell, A.S. 1942. The oceanic Tintinnoina of the plankton gathered during the last cruise of the Carnegie. Scient. Res. Cruise VII Carnegie during 1928-1929 under the command of Captain J. P. Ault. Carnegie Institute Washington Publications, n. 537, Washington, 162p.

Claparéde, E. \& J. Laackmann. 1858. Études sur les infusoires et les rhizopodes. Memoires des Institute Genevois, Paris, 5 (3): $1-260$.

Cleve, P.T. 1900. Some Atlantic Tintinnodea. Kongliga Svenska Vetenskaps Akademies Handlingar, Stockholm, 56: 969-975.

Cordeiro, T.A.; F.P. Brandini \& P. Martens. 1997. Spatial distribution of the Tintinnina (Ciliophora, Protista) in the North Sea, spring of 1986. Journal of Plankton Research, Oxford, 19 (10): 1371-1383.

DADAY, E. 1887. Monographie der Familie der Tintinnoideen. Mittlerung Zoological Statione Neapel, Budapeste, 7: 1-172.

DhN. 1989. Operação Monitor IV. Rio de Janeiro, DHN, Marinha do Brasil, 22p.

Dodson, A.N. \& W.H. Thomas. 1978. Reverse filtration, p. 104-107. In: A. Sournia (Ed.). Phytoplankton manual. Monographs on oceanographic methodology 6. Paris, UNESCO, 300p.

EhrenberG, C.G. 1854. Das organische Leben des Meeresgrundes in bis 10,800 und 12,000 Fuss Tiefe. Monatsberichte der Koniglisch Preussischen Akademie der Wissenschaften zu Berlin: 54-76.

EnTZ, Sr., G. 1884. Ueber Infusorien des Golfes von Neapel. Mittheillungen Zoological Statione Neapel, Budapest, 5: 289-444.

. 1885. Zur näheren der Tintinnoden. Mittheillungen Zoological Statione Neapel, Budapest, 6: 185-216.

Fernandes, L.F. 1999. Tintininos (Protozoa-Ciliophora-Subordem Tintinnina) de águas subantárticas e antárticas entre a Argentina e a Península Antártica $\left(35^{\circ} \mathrm{S}-62^{\circ} \mathrm{S}\right.$ ) (Novembro de 1992). Revista Brasileira de Oceanografia, São Paulo, 47 (2): 155-171.

— 2004. Tintininos (Ciliophora, Tintinnina) de águas subtropicais na região Sueste-Sul do Brasil. I. Famílias Codonellidae, Codonellopsidae, Coxliellidae, Cytarocyllidae, Epiplocylidae, Petalotrichidae, Ptychocylidae, Tintinnididae e Undellidae. Revista Brasileira de Zoologia, Curitiba, 21 (3): 551-576.

Fernandes, L.F. \& F.P. Brandini. 1999. Comunidades micro- 
planctônicas no Oceano Atlântico Sul Ocidental: biomassa e distribuição em novembro de 1992. Revista Brasileira de Oceanografia, São Paulo, 47 (2): 189-205.

Garrison, D.L. \& M.M. Gowing. 1993. Protozooplankton, p.123165. In: E.I. Friedmann (Ed.). Antarctic microbiology. London, Wiley-Liss, 800p.

Emílsson, I. 1961. The shelf and coastal waters off the Southern Brazil. Boletim do Instituto oceanográfico da Universidade de São Paulo, São Paulo, 11 (2): 101-112.

HADA, Y. 1938. Studies on the Tintinnoinea from the Western Tropical Pacific. Journal of the Faculty of Sciences Hokkaido Imperial University, Series VI, Zoology, Hokkaido, 6 (2): 87-190.

- 1970. The protozoan plankton of the Antartic and Subantartic seas. Japanese Antartic Research Expedition Scientific Report, Series E, no. 3, Tokyo, 51p.

HaEckel, E. 1873. Ueber einige neue pelagische Infusirien. Jenaische Zeitschrift für Naturwissenschaft, Jena, 7: 561-568.

Jörgensen, E. 1924. Mediterranean Tintinnidae. Report of Danish Oceanographic Expedition 1908-1910 to the Mediterranean and adjacent seas, Kopenhagen, 2: 1-114.

Kimor, B. \& B. Golandsky-Baras. 1981. Distribution and ecology of the tintinnids in the gulf of Elat (Aqaba), Red sea. Journal of Plankton Research, Bristol, 3 (3): 445-459.

Kofoid, C.A. \& A.S. Campbell. 1929. A conspectus of the marine and freshwater Ciliata belonging to the suborder Tintinnoinea, with descriptions of news species principally from the Agassiz Expedition to the Eastern tropical Pacific, 19041905. University of California Publications in Zoology, Los Angeles, 34: 1-403.

1939. Reports on the scientific results of the expedition to the Eastern tropical Pacific, in charge to Alexander Agassiz, by U.S. Fish commision steamer "Albatross", from October, 1904, to March, 1905, Lieut.-Commander L.M. Garrett, U.S.N. commanding. XXXVII. The Ciliata: The Tintinnoinea. Bulletin of the Museum of Comparative Zoology of Harvard College, Harvard, 85: 1-473.

Krsnic, F. 1987. On the ecology of tintinnines in the bay of Mali Stone (Eastern Adriatic). Estuarine Coastal and Shelf Science, London, 24: 401-418.

. 1988. The family Xystonellidae (Ciliophora Tintinnina) in the Adriatic sea. Journal of Plankton Research, Oxford, 10 (3): 412-429.

LAACKMANN, H. 1907. Antarktische Tintinnen. Zoologischer Anzeiger, Berlin, 31: 235-239.

- 1910. Die Tintinnodeen der deutschen Südpolarexpedition 1901-1903. Deutsche Südpolar- expedition, Berlin, 11: 340-496.

Levine, N.D.; J.O. Corliss; F.E.G. Cox; G. Deroux; J. Grain; B.M. Honiberg; G.F. LeEdale; A.R.III Loeblich; J. Lom; D. LynN; E.G. Merinfeld; F.C. Page; G. Poljansky; V. Sprague; J. Vavra \& F.G. Wallace. 1980. A newly revised classification of the Protozoa. Journal of Protozoology, Sttutgart, 27 (1): 37-58.
MatsuUra,Y. 1986. Contribuição ao estudo da estrutura oceanográfica entre Cabo Frio (RJ) e Cabo de Santa Marta Grande (SC). Ciência e Cultura, São Paulo, 38 (8): 1439-1450.

Ostenfeld, C.H. 1899. "Plankton" in Iagttagelser over Overfladevandets temperatur Salt-holdighed og plankton paa islandske og Grönlandske skibsrouter i 1898, af C. F. Wandel bearbjdede af Martin Knudsen of C. Ostenfeld (Kjöbenhavn, Gad), p. 47-93.

Pierce, R.W. \& J.T. Turner. 1993. Global biogeography of marine tintinnids. Marine Ecology Progressive Series, Kiel, 94: 11-26.

SASsI, R. \& G.N. MeLo. 1986. Tintinnina (Protozoa - Ciliophora - Oligotrichida) from the first brazilian expedition to the Antartic. Anais da Academia Brasileira de Ciências, Rio de Janeiro, 58 (Supl.): 63-83.

- 1989. Hyaline Tintinnina (Protozoa - Ciliophora Oligotrichida) from northeastern Brazilian coastal reefs. Boletim do Instituto Oceanográfico, São Paulo, 37 (1): 59-74.

Schmidt, J. 1901. Some Tintinnodea from the Gulf of Siam. Videnskabellige Meddeleljer fra den Natur Historiske Forening i Kjobenhaun, 1901, Kopenhagen, p. 183-190.

Sournia, A. 1982. Is there a shade flora in the marine plankton? Journal of Plankton Research, Oxford, 4 (2): 391-399.

Sousa E SiLva, E. 1950. Les tintinnides de la Baie de Cascais (Portugal). Bulletin des Institute Océanographique du Monaco, Paris, 47 (979): 1-28.

- 1954. Tintinnoinea do plâncton marinho de Angola. Estudos de Biologia Marítima, Anais, Lisboa, 9 (2): 179 243.

Souto, S. 1970a. Tintinidos de la costa atlántica entre los $31^{\circ} \mathrm{y}$ $35^{\circ}$ de latitud Sur (Uruguay y Sur de Brasil). Physis, Buenos Aires, 30: 187-208.

- 1970b. Tintinidos de la costa brasilera colectados por el Walther Herwig. Physis, Buenos Aires, 30: 209-224. - 1981. Tintinnina, p. 303-381. In: D. Boltovskoy (Ed.). Atlas del Zooplancton del Atlântico Sudoccidental y metodos de trabajo con el zooplancton marino. Mar del Plata, Publicación Especial INIDEP, 1000p.

STRAND, 1926. Miscellanea nomenclatorica zoologica et paleontologica i-ii. Archiv für Naturgeschichte, Berlin, 92A (8): 30-75. XCII, A: 30.

Thompson, G.A.; V.A. Alder; D. Boltovskoy \& F.P. Brandini. 1999. Abundance and biogeography of tintinnids (Ciliophora) and associated microplankton in the Southwestern Atlantic Ocean. Journal of Plankton Research, Oxford, 21 (7): 1265-1298.

VALENTIN, J.L. 1989. A dinâmica do plâncton na ressurgência de Cabo Frio - R.J., p. 25-35. In: F.P. BRANDINI (Ed.). Memórias do III Encontro Brasileiro de Plâncton. Curitiba, 100p.

VENRICK, E.L. 1988. The vertical distributions of chlorophyll and phytoplankton species in the North Pacific central environment. Journal of Plankton Research, Oxford, 10 (5): 987-998.

Recebido em 11.VI.2003; aceito em 28.VII.2004. 